\title{
FastMix: A Versatile Multi-Omics Data Integration Pipeline for Cell Type-Specific Biomarker Inference
}

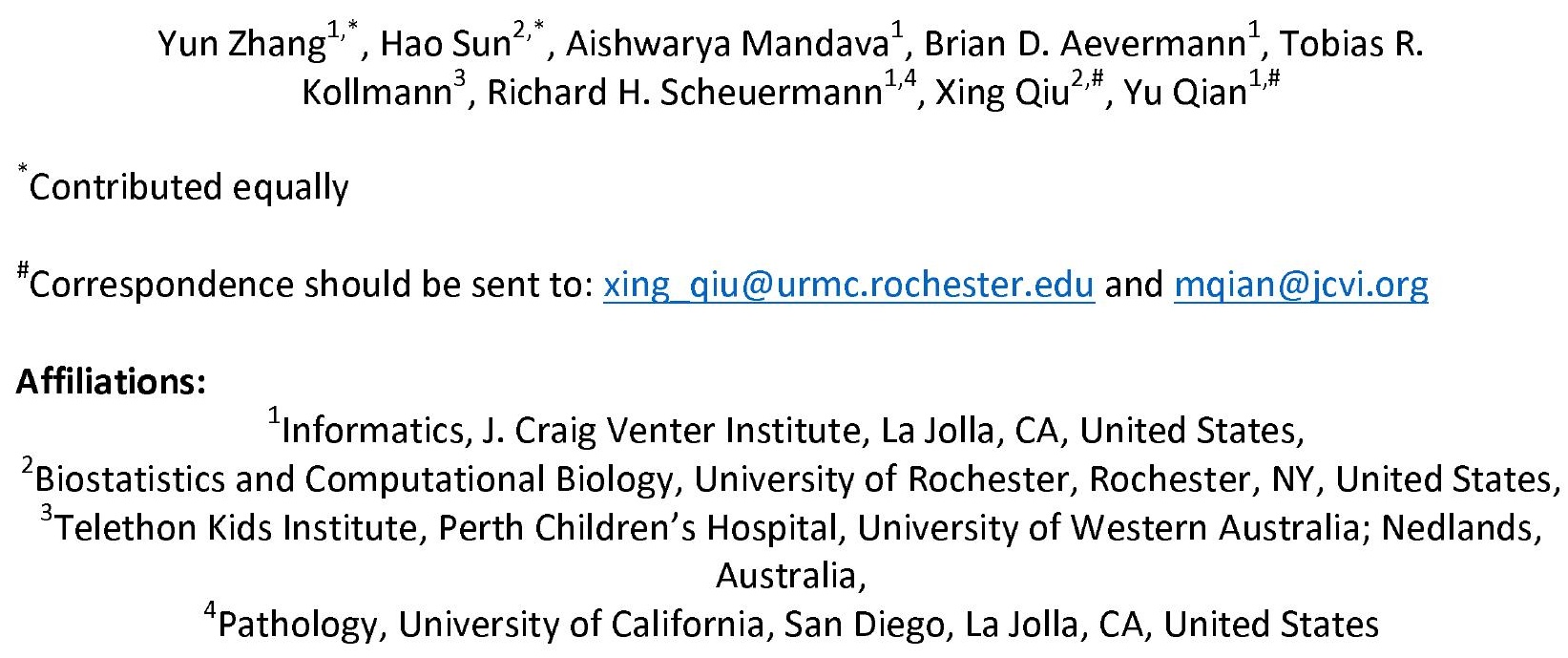

\begin{abstract}
We developed a novel analytic pipeline - FastMix - to integrate flow cytometry, bulk transcriptomics, and clinical covariates for statistical inference of cell type-specific gene expression signatures. FastMix addresses the "large $p$, small $n$ " problem via a carefully designed linear mixed effects model (LMER), which is applicable for both cross-sectional and longitudinal studies. With a novel moment-based estimator, FastMix runs and converges much faster than competing methods for big data analytics. The pipeline also includes a cuttingedge flow cytometry data analysis method for identifying cell population proportions. Simulation studies showed that FastMix produced smaller type I/II errors with more accurate parameter estimation than competing methods. When applied to real transcriptomics and flow cytometry data in two vaccine studies, FastMix-identified cell type-specific signatures were largely consistent with those obtained from the single cell RNA-seq data, with some unique interesting findings.
\end{abstract}


medRxiv preprint doi: https://doi.org/10.1101/2021.09.14.21263182; this version posted September 27, 2021. The copyright holder for this

preprint (which was not certified by peer review) is the author/funder, who has granted medRxiv a license to display the preprint in perpetuity.

It is made available under a CC-BY-NC-ND 4.0 International license .

Introduction

31 High throughput multi-omics technologies are becoming popular. In a multi-omics study,

32 different types of sample characteristics of the same subject, e.g., genomics, transcriptomics,

33 epigenomics, proteomics and metabolomics, are measured using a variety of bioassays. Recent

34 publications [1-7] have shown that systems biology approaches based on multi-omics

35 integrative data analysis can effectively identify important patterns that otherwise would be

36 missed using a single assay. One key challenge for multi-omics data integration is the "large $p$,

37 small $n$ " problem. Each type of assay can measure many analytes. As a result, the total number

38 of experiment variables $(p)$ involved in a multi-omics study is usually large. The number of

39 experimental subjects and their samples $(n)$, however, is usually more limited due to cost and

40 enrollment capability. When $p>n$, it is unreliable to infer or interpret the relationship among

41 the variables using standard regression models.

42 Dimensionality reduction and regularization are two common approaches to address this issue.

43 Common data dimensionality reduction techniques include linear projection methods such as

44 principal component analysis (PCA) [8], canonical correlation analysis (CCA) [9] and partial least

45 squares (PLS) [10], as well as non-linear embedding methods such as t-distributed stochastic

46 neighbor embedding (t-SNE) [11] and uniform manifold approximation projection (UMAP) [12].

47 Well-established regularization methods include ridge [13], LASSO [14], and elastic-net [15].

48 Currently, major efforts in the field of multi-omics integrative analysis focus on using one or

49 both types of approaches to address the "large $p$ " problem. For example, DIABLO [16] uses

50 sparse generalized canonical correlation analysis (sGCCA) with LASSO penalty to integrate data 
medRxiv preprint doi: https://doi.org/10.1101/2021.09.14.21263182; this version posted September 27, 2021. The copyright holder for this

preprint (which was not certified by peer review) is the author/funder, who has granted medRxiv a license to display the preprint in perpetuity.

It is made available under a CC-BY-NC-ND 4.0 International license .

51 from multiple omics assays and predict patient's disease type. LUCID [17] uses a joint

52 probabilistic model with latent variables for integrated clustering regularized by LASSO. In the

53 emerging single cell genomics field, UMAP and other embedding techniques are frequently

54 used for the dimensionality reduction purpose for multi-modality data integration $[18,19]$.

55 However, the fundamental question of biomarker detection, which requires the integration of

56 both assay data and clinical covariates with differential analysis, has not been solved.

57 Among the applicable statistical models, linear-mixed effects regression (LMER) is a powerful

58 and generalizable framework that can be used to address the "large $p$, small $n$ " problem for

59 multi-omics data integration. Regularized fixed effects regression models have been shown to

60 solve the "large $p$, small $n$ " issue and reduce the variability in the estimation procedure by

61 shrinking the estimates toward zero. On the other hand, LMER shrinks the estimates toward the

62 fixed effects instead of zero, so that they have less variability and are less biased [20, 21].

63 However, LMER is not widely applied to high-throughput multi-omics data because of its high

64 computational cost and numerical instability. Conventionally, a LMER model is solved by an

65 expectation-maximization (EM) algorithm, which iteratively finds maximum likelihood estimate

66 of regression parameters [22]. This iterative process is slow and prone to convergence issues,

67 which makes the LMER almost impossible to be applied to analyze data from high-throughput

68 studies.

69 To reduce the high computational cost of the iterative EM algorithm, we designed a non-

70 iterative, moment-based covariance estimator, which is not only more robust than the iterative

71 EM process but also more efficient, requiring a small fraction of EM's run time. To both 
medRxiv preprint doi: https://doi.org/10.1101/2021.09.14.21263182; this version posted September 27, 2021. The copyright holder for this

preprint (which was not certified by peer review) is the author/funder, who has granted medRxiv a license to display the preprint in perpetuity.

It is made available under a CC-BY-NC-ND 4.0 International license .

72 demonstrate the utility and evaluate the performance of the proposed approach, we combined

73 the moment-based estimation of LMER together with downstream differential expression (DE)

74 analysis into a computational pipeline for inferring cell type-specific differentially expressed

75 genes (DEGs) from bulk gene expressions and flow cytometry (FCM) data, a problem that is

76 commonly encountered in immunology studies but not specifically addressed by the existing

77 multi-omics data integration methods. As shown in Figure 1a, the proposed model-FastMix

78 - takes in three sets of input data: (i) bulk gene expressions measured by microarray or RNA

79 sequencing; (ii) proportions of cell populations identified from FCM data; and (iii) experiment

80 covariates and clinical parameters such as demographics, cohorts, and visits of the subjects.

81 Figure 1b depicts the main steps in the FastMix modeling and testing framework and key

82 techniques to obtain accurate parameter estimations. Expected improvement of parameter

83 estimation by the FastMix is illustrated in Figure $1 \mathrm{c}$.

84 FastMix optimizes the bias-variance tradeoff in a way that the DEGs (dots in black) can be

85 estimated closer to the ground truth than the standard approach (Figure 1c). Figure 1d provides

86 a schematic representation of the whole analytic pipeline. Unlike traditional unsupervised

87 analyses that rely on predefined marker genes (which are often incomplete or totally unknown

88 when the cell types are novel) to define cell types for estimating the cell composition data, our

89 proposed pipeline integrates the FCM data with bulk gene expression data to supervise the cell

90 type-specific inference. FastMix inference provides a baseline method for cell type-specific

91 data analysis to complement the cutting-edge single cell transcriptomics assay which still needs

92 time to be fully mature and widely affordable. 
medRxiv preprint doi: https://doi.org/10.1101/2021.09.14.21263182; this version posted September 27, 2021. The copyright holder for this

preprint (which was not certified by peer review) is the author/funder, who has granted medRxiv a license to display the preprint in perpetuity.

It is made available under a CC-BY-NC-ND 4.0 International license .

93 In addition, the pipeline depicted in Figure $1 \mathrm{~d}$ addresses the analysis of FCM data by including a

94 cutting-edge computational method - DAFi [23] - to identify composition/proportions of the

95 cell populations. It makes the pipeline advantageous over the existing data integration

96 approaches when the scientific study includes FCM assay data. The DAFi-based analysis

97 improves not only the reproducibility of the FCM data analysis but also the accuracy of the

98 proportions of the cell populations for improving the downstream FastMix inference. It is

99 important to note that the pipeline depicted in Figure 1d can be applied to analyze other types

100 of multimodal datasets for compositional and bulk profiling integrative analysis. For example,

101 multimodal data from metagenomics and metabolomics assays commonly include bulk analysis

102 and composition of microbial communities for which FastMix can be applied to identify the

103 community-specific biomarkers. FastMix is freely accessible as an open source package at

104 https://github.com/terrysun0302/FastMix.

105 isResults

106 Fast unfolding of cell type mixture by integrating multimodal omics data

107 FastMix is primarily designed to integrate two popular assays - flow cytometry and

108 transcriptome profiling - in multi-omics studies. In this scenario, FastMix takes in three sets

109 of input data: (i) clinical covariates (denoted as Clin), such as age, sex, treatment group, (ii) cell

110 type proportions measured by flow cytometry assays on heterogeneous populations (denoted

111 as Cell), and (iii) bulk gene expression measured by microarray or RNA sequencing (denoted as

$112 Y ; Y$ is a sample-by-gene matrix following the regression model convention). Without

113 FastMix, separate regression models can be used to quantify the linear associations between 
medRxiv preprint doi: https://doi.org/10.1101/2021.09.14.21263182; this version posted September 27, 2021. The copyright holder for this preprint (which was not certified by peer review) is the author/funder, who has granted medRxiv a license to display the preprint in perpetuity.

It is made available under a CC-BY-NC-ND 4.0 International license .

114 two out of the three data inputs, i.e., associations between $Y$ and $\mathrm{Clin}$, associations between $Y$

115 and Cell, or associations between Cell and Clin. FastMix simultaneously studies the

116 associations between all three sets of variables in the following unified linear regression model

$$
Y=X W+E
$$

117 where $X$ is a three-component design matrix, $X:=($ Cell Clin Cell $\times$ Clin $), W$ is a matrix of

118 regression coefficients (weights) to be estimated, and $E$ is a matrix of errors. By including the

119 interaction term Cell $\times$ Clin, FastMix makes cell type-specific inferences beyond the bulk

120 level analysis. Typically, the above model is an under-determined system. FastMix reduces

121 the model complexity by using the linear mixed effects regression (LMER) techniques. It

122 introduces the gene-specific mixed effects, such that $\beta_{l i}=\beta_{l}+\gamma_{l i}$, where $\beta_{l}$ is the fixed effect

123 of the $l$ th covariate to the entire transcriptome, and $\gamma_{l i}$ is the gene-specific random effect of

124 the $i$ th gene. FastMix also provides a computational algorithm that is much faster than the

125 traditional expectation-maximization (EM) algorithm to solve large-scale LMER model with

126 high-throughput data. The FastMix algorithm is a non-iterative procedure that uses a novel

127 moment-based estimator for the covariance matrix of random effects (denoted as $\hat{B}_{T}$ ). Of note,

128 FastMix estimates the covariance matrix with outlier trimming and bias correction, therefore

129 it is robust to numerical aberrations induced by outliers and DEGs in the data. Estimates of the

130 fixed effects $\left(\hat{\beta}_{l}\right)$ and random effects $\left(\hat{\gamma}_{l i}\right)$ can then be computed using the weighted least

131 squares (WLS) and empirical best linear unbiased predictor (EBLUP) techniques. (See Figure 1b

132 and the Methods section for more details.) 
medRxiv preprint doi: https://doi.org/10.1101/2021.09.14.21263182; this version posted September 27, 2021. The copyright holder for this preprint (which was not certified by peer review) is the author/funder, who has granted medRxiv a license to display the preprint in perpetuity.

It is made available under a CC-BY-NC-ND 4.0 International license .

133 The overarching goal of the FastMix model is to perform DE analyses with respect to the

134 components in the design matrix. Though no classical hypothesis test can be applied to the

135 random effects for theoretical reasons; in practice, FastMix introduces a novel competitive

136 test with quasi-p-value to identify DEGs that have significantly larger or smaller predicted

137 random effects $\hat{\gamma}_{l i}$ (i.e., cell type-specific effects) to practically rank the importance of genes in

138 the whole transcriptome. To this end, FastMix incorporates a DEG indicator, and assigns the

139 random effects a mixture distribution conditional on the DEG indicator based on empirical

140 Bayes method [24, 25]. For each component of the design matrix, FastMix inference on

141 random effects can be interpreted as:

- Cell - detection of cell type signature genes that distinguish cell types from each other,

143 - Clin - bulk-level gene expression differential analysis,

144 - Cell $\times$ Clin - cell type-specific differential analysis, i.e., cell type-specific DEGs.

145 Note that FastMix is able to incorporate arbitrary weight matrices at the sample level, which

146 can be used to account for the serial correlation in longitudinal studies. We will provide some

147 practical guidance on how to construct such a weight matrix in the Methods section. We refer

148 to this model as the weighted FastMix model. FastMix with known weights is shown to

149 perform better than FastMix without weight if the data are known to fail the independent

150 and identically distributed assumption (please see Supplementary Material, Section 2.3 and 3.5).

151 In the rest of this section, we illustrated the properties of FastMix in extensive simulation

152 studies. In two real data studies, we applied (weighted) FastMix to carry out cell type-specific 
medRxiv preprint doi: https://doi.org/10.1101/2021.09.14.21263182; this version posted September 27, 2021. The copyright holder for this preprint (which was not certified by peer review) is the author/funder, who has granted medRxiv a license to display the preprint in perpetuity. It is made available under a CC-BY-NC-ND 4.0 International license .

153 inference with a focus on neutrophils in a hepatitis B virus (HBV) vaccine study and a focus on

154 lymphocytes in an influenza infection study. We chose these two cell populations because

155 neutrophils play important roles in pathogenesis of liver diseases and immune responses to

156 HBV vaccines [26, 27]. Also, influenza infection is known to be associated with a relative

157 lymphopenia/neutrophia ratio [28]. 
medRxiv preprint doi: https://doi.org/10.1101/2021.09.14.21263182; this version posted September 27, 2021. The copyright holder for this preprint (which was not certified by peer review) is the author/funder, who has granted medRxiv a license to display the preprint in perpetuity.

It is made available under a CC-BY-NC-ND 4.0 International license .

\section{Simulation I: the effect of trimming and robustness estimation of covariance matrix}

159 We designed simulation I to illustrate the advantage of the moment-based covariance matrix

160 estimator. For illustration purpose, we considered two cell types, namely Cell1 and Cell2, whose

161 random effects are $\gamma_{1 i}$ and $\gamma_{2 i}$, respectively. Figure 1c shows the effect of the proposed

162 embedded robust covariance estimator $\widehat{B}_{T}$ (green ellipse) for estimating the true covariance

163 matrix between the random effects $B$ (black ellipse). In the simulation, we generated 5000

164 genes (dots); among them, 250 genes were true DEGs (black dots) in the direction of Cell1, i.e.,

165 Cell1-specific DEGs. Note that the existence of true DEGs can be considered as outliers under

166 the null hypothesis $[29,30]$ that may lead to over-estimation of the covariance matrix. In the

167 estimation procedure of FastMix, an initial covariance estimator (red ellipse) was constructed

168 first using the ordinary least squares (OLS) regression technique (see the Methods section),

169 which is non-robust to bias caused by DEGs. Trimming and bias correction techniques were

170 then applied to re-estimate the initial estimated covariance matrix. Simulation I showed that

171 the final estimator $\widehat{B}_{T}$ was robust to the existence of outliers (DEGs), and accurately

172 recapitulated the true covariance matrix, i.e., the overlay of the green ellipse and the black

173 ellipse.

174 Simulation II: comparing performance of FastMix with other regression models

175 Simulation II was conducted to verify the statistical and computational properties of the

176 proposed method. We generated synthetic gene expression values for 5000 genes and 50

177 subjects. For each subject, we generated three cell proportions (Cell1, Cell2, and Cell3), one

178 continues clinical covariate (Severity), and one categorical clinical covariate (Sex). In the 
medRxiv preprint doi: https://doi.org/10.1101/2021.09.14.21263182; this version posted September 27, 2021. The copyright holder for this preprint (which was not certified by peer review) is the author/funder, who has granted medRxiv a license to display the preprint in perpetuity.

It is made available under a CC-BY-NC-ND 4.0 International license .

179 simulation design, four scenarios were considered: with or without true DEGs, and with or

180 without correlation between random effects. Simulation details are described in the Methods

181 Section.

182 Here, we systematically evaluated the computational efficiency and accuracy for estimating $B$,

183 the covariance matrix of random effects. The FastMix model is a special case of LMER, with

184 robust estimation of $B$ and bias-correction procedure for fixed effect. The standard

185 implementation of LMER in $\mathrm{R}$ is the Ime4 package, which uses an iterative expectation-

186 maximization (EM) algorithm to obtain the maximum likelihood estimator for $B$. The Ime4

187 implementation is very time-consuming for estimating the full covariance matrix $B$. In practice,

188 users may specify an independent correlation structure, i.e., assuming no correlation between

189 random effects thus $B$ is diagonal. The Ime4 implementation with independent assumption

190 (Ime4_ind) is more efficient than the default Ime4 implementation since much simpler

191 covariance structure is assumed in the model. Similarly, we also implemented the independent

192 assumption for the random effects in the FastMix algorithm (FastMix_ind). For the

193 completeness of comparison, we reported the time consumed in seconds and mean square

194 error (MSE) for estimating $B$ using Ime4_ind, Ime4, FastMix, and FastMix_ind under the

195 four simulated scenarios in Table 1a.

196 Table 1a showed that, when the random effects were independent and without DEGs,

197 Ime4_ind was the theoretically best approach and had the lowest MSE. While the accuracy of

198 Ime4_ind and FastMix_ind were both at the minimal level $(\mathrm{MSE}=0.02$ and MSE $=0.04$,

199 respectively), FastMix_ind used only $2 \%$ of the computational time of Ime4_ind. When the 
medRxiv preprint doi: https://doi.org/10.1101/2021.09.14.21263182; this version posted September 27, 2021. The copyright holder for this

preprint (which was not certified by peer review) is the author/funder, who has granted medRxiv a license to display the preprint in perpetuity.

It is made available under a CC-BY-NC-ND 4.0 International license .

200 random effects were correlated and without DEGs, FastMix had the smallest MSE (0.21) and

201 was more than 300 times faster than the full-pledged Ime4 algorithm, which had the second

202 best MSE (0.32). For the simulation scenarios with DEGs, the FastMix implementations were

203 always (with or without correlation) the best performers with the smallest MSEs and used tiny

204 amount of computational time. The Ime4-based approaches were not able to obtain accurate

205 estimates, because the maximum likelihood estimator used in Ime4 was not robust to effects

206 introduced by DEGs (outliers). On the other hand, FastMix was robust to these effects due to

207 the use of trimming. In Supplementary Material, sections 3.2 and 3.3, we also showed that

208 FastMix greatly reduced the bias in the fixed effect estimation compared to the Ime4

209 approach and other robust covariance estimators [31-33]. Among all the methods compared,

210 FastMix had the most robust performance (Supplementary Material, Tables 1 and 3).

211 Next, we compared FastMix with ordinary least square (OLS) and Ridge regression for

212 regression coefficient estimation. One primary reason to use LMER instead of the standard OLS

213 regression is to reduce the variability of the estimated regression coefficients. For the same

214 purpose, Ridge regression is also well-known for stabilizing the regression coefficients using

215 regularization. In the second simulation, we compared the accuracy of estimating the gene-

216 specific linear coefficients $\beta_{l i}$, using FastMix, OLS, and Ridge regressions. We considered the

217 most real simulation scenario, i.e., with correlation and DEGs, for this evaluation. The

218 regularization parameter in ridge regression was selected by the generalized cross-validation

219 (GCV) criterion. Table 1b showed that, FastMix had the smallest total MSE among the three

220 compared methods. In this simulation, the standard deviations of FastMix and Ridge (0.017

221 and 0.046, respectively) were both much smaller than that of OLS (0.2), suggesting that both 
medRxiv preprint doi: https://doi.org/10.1101/2021.09.14.21263182; this version posted September 27, 2021. The copyright holder for this

preprint (which was not certified by peer review) is the author/funder, who has granted medRxiv a license to display the preprint in perpetuity.

It is made available under a CC-BY-NC-ND 4.0 International license .

222 methods could achieve the shrinkage effect, i.e., stabilizing the regression coefficients. A closer

223 look at the biases of individual coefficients suggested that the FastMix and OLS estimates

224 could be regarded as practically unbiased. On the other hand, estimates of Ridge regression had

225 large bias, because the $L^{2}$ regularization in Ridge regression shrank the estimates toward zero

226 [13], not the fixed effects.

227 Simulation III: Comparing FastMix with existing cell type-specific differential analysis

228 method

229 Shen-Orr et al. proposed csSAM [34] - a cell type-specific differential analysis method for

230 heterogeneous biological samples using gene expression data and relative cell type frequencies.

231 cSSAM is also a regression-based model solved by the standard OLS technique; and the

232 differential analysis is conducted by the established SAM [35] pipeline for bulk gene expression.

233 A major limitation of cSSAM is that it only performs two-group comparison, i.e., one binary

234 covariate. For a fair comparison, we redesigned a simpler simulation study for the same

235 number of genes and subjects, which had three cell proportions (Cell1, Cell2, and Cell3) and one

236 binary covariate (Group), to compare the type I error rate, power, and computational time for

237 cell type-specific DEG detection using FastMix and csSAM. By design, there were true cell

238 type-specific DEGs for Cell1 and Cell2, but not Cell3; two scenarios with and without correlation

239 between random effects were also considered. Simulation details are described in the Methods

240 Section.

241 Table 1c-e showed the simulation performance of the two cell type-specific methods. In both

242 correlation and no correlation scenarios, FastMix had acceptable type-I error rate $(5 \%$ 7\%), 
medRxiv preprint doi: https://doi.org/10.1101/2021.09.14.21263182; this version posted September 27, 2021. The copyright holder for this preprint (which was not certified by peer review) is the author/funder, who has granted medRxiv a license to display the preprint in perpetuity.

It is made available under a CC-BY-NC-ND 4.0 International license.

243 while csSAM had much higher type-I error rate than FastMix in all cases (Table 1c); FastMix

244 also had better statistical power (on average 65\%) for detecting Cell1-specific and Cell2-specific

245 true DEGs than csSAM (on average 50\%) in the simulation (Table 1d). Overall, FastMix not

246 only showed superior performance in the simulation results, but also used just 1/10 of the

247 computational time of csSAM (Table 1e).

248 In Supplementary Material, Section 3.4, we showed that when there were more up-regulated

249 DEGs and fewer down-regulated DEGs in a slightly different simulation, similar patterns of the

250 type-I error, statistical power, and computational efficiency performance were observed for

251 both methods as those shown in Table 1c-e.

252 FastMix multi-omics integration reveals consistent cell type-specific signature genes with

253 scRNA-seq technology

254 We applied FastMix to a multimodal study that investigates immune responses to the

255 licensed hepatitis B vaccine - Engerix-B - for the Human Vaccine Project (HVP) [36]. The HVP01

256 study [37] contains well used assays on whole blood or peripheral blood mononuclear cell

257 (PBMC) samples from adults with wide age range (40-80 years old), including flow cytometry for

258 immunophenotyping, RNA-seq for bulk transcriptomics, and virus neutralization assay for

259 serum antibody titers (anti-HBs). In addition, this study also has scRNA-seq data for immune

260 cells using the Smart-Seq2 [38] protocol, which we would use as the ground truth to validate

261 our FastMix results. 
medRxiv preprint doi: https://doi.org/10.1101/2021.09.14.21263182; this version posted September 27, 2021. The copyright holder for this preprint (which was not certified by peer review) is the author/funder, who has granted medRxiv a license to display the preprint in perpetuity.

It is made available under a CC-BY-NC-ND 4.0 International license .

262 Engerix-B requires three doses to reach clinically proven immune protection [39]. In the HVP01

263 study, there are 15 subjects. After Dose 3, all subjects responded to vaccination, but some had

264 much higher immune protection measured by the anti-HBs titer than others (Supplementary

265 Figure S1). We grouped subjects who had anti-HBs titer $>5000 \mathrm{mUI} / \mathrm{mL}$ after Dose 3 as high

266 responders (5 subjects), and otherwise low responders (10 subjects). Immunophenotyping by

267 flow cytometry and gene expression by RNA-seq of whole blood and single immune cells were

268 collected at 5 time points (Day 0,1,3,7, and 14). Based on the markers used in the flow

269 cytometry panels, we identified the abundant neutrophils (CD45+CD66+), non-neutrophils

270 (CD45+ CD66-), and rest populations (Figure 2a) following the DAFi gating hierarchy [23] (see

271 the Methods section). Using all time points, we fitted a weighted FastMix model for the bulk

272 RNA-seq gene expression with a design matrix of cell proportions, clinical covariates including

273 response group and age (Supplementary Figure S1), and their interactions.

274 First, we looked at the DEG list of the main terms, which can be interpreted as the signature

275 genes of each cell population. Broadly speaking, these signature genes are differentially

276 expressed in the specific cell population when compared with the rest of the cell populations.

277 Out of the 13,157 genes available in the processed bulk RNA-seq data, FastMix identified 851

278 signature genes for the neutrophil population, 520 signature genes for the non-neutrophil

279 population, and 30 signature genes for the rest population. Because the rest population is the

280 most heterogenous population, we would expect that not many signature genes could be

281 identified for the rest population that contained a mixture of cell types. 
medRxiv preprint doi: https://doi.org/10.1101/2021.09.14.21263182; this version posted September 27, 2021. The copyright holder for this preprint (which was not certified by peer review) is the author/funder, who has granted medRxiv a license to display the preprint in perpetuity.

It is made available under a CC-BY-NC-ND 4.0 International license .

282 To validate the FastMix signature genes for the well-defined cell population (i.e., neutrophils),

283 we used a completely independent assay data from the unbiased scRNA-seq whole

284 transcriptome expression profiling. We followed a standard scRNA-seq analysis pipeline

285 including low-dimensional embedding of cells on UMAP [40] with cell clusters color labeled by

286 the ground truth cell types based on cell surface markers in FCM sorting, and scRNA-seq DE

287 analysis by a nonparametric hypothesis testing approach [41] for cell type DE gene detection

288 for scRNA-seq data, which were conceptually comparable with the signature genes detected by

289 FastMix. UMAP visualization of the ground truth cell types (Figure $2 b$ ) showed a good

290 separation of the neutrophil population from other cell populations. We applied the scRNA-seq

291 DE analysis to detect DEGs between the neutrophils and all other cells, which identified 2,744

292 neutrophil cell type DE (a.k.a. signature) genes from 58,036 annotated genes in total.

293 We compared the FastMix and scRNA-seq results of neutrophil signature genes in Figure 2c.

294 The majority (>50\%) of the FastMix signature genes overlapped with the scRNA-seq signature

295 genes. Specifically, $72 \%$ of the top 100 FastMix signature genes were consistent with the

296 scRNA-seq signature genes. The overlapping rate gradually decreased as we included more top

297 genes in the comparison, meaning that FastMix ranked more "ground truth" (scRNA-seq)

298 signature genes at the top in its DEG list. For pragmatic use, we further selected 365 scRNA-seq

299 signature genes that have substantial fold change $(\mathrm{FC})$, i.e., $|\log F C|>1$. The Venn diagram

300 (Figure 2d) showed that 39 of the top 100 FastMix signature genes were overlapped with the

301 selected scRNA-seq signature gene list, suggesting that the FastMix signature genes were not

302 only close to the scRNA-seq ground truth, but also contained more practically useful genes in

303 the top ranked genes ( $16 \%$ of the scRNA-seq signature genes passed the logFC threshold vs. $39 \%$ 
medRxiv preprint doi: https://doi.org/10.1101/2021.09.14.21263182; this version posted September 27, 2021. The copyright holder for this preprint (which was not certified by peer review) is the author/funder, who has granted medRxiv a license to display the preprint in perpetuity.

It is made available under a CC-BY-NC-ND 4.0 International license .

304 of the top 100 FastMix signature genes passed the logFC threshold). Among the 39 common

305 genes, many of them are highly relevant to both neutrophils and Hepatitis $\mathrm{B}$, e.g., CXCR1/2

306 plays an important role [26] in hepatic inflammatory response [42]. The same can be seen for

307 the interferon-induced proteins from IFIT and IFITM family genes (the 39 genes include IFIT2,

308 IFITM2, IFITM3, etc.) Furthermore, we plotted the scRNA-seq expression values of the 39

309 common signature genes across all cell types (Figure 3a), compared with the bottom genes

310 (Figure 3b) and top (Figure 3c) genes identified by FastMix in violin plots. It is clear to see

311 abundant gene expression in the neutrophils for common and top FastMix signature genes,

312 but almost no expression in the bottom genes.

313 Identifying cell type-specific interferon signaling pathway genes after Hepatitis B vaccination

314 using FastMix

315 Next, we compared FastMix and csSAM for identifying the cell type-specific DEGs. With the

316 above FastMix model of HVP01 study, we focused our comparison on the neutrophil-specific

317 DEGs with respect to the response group (since csSAM only performs two-group DE analysis for

318 the cell type-specific SAM model). FastMix identified 495 neutrophil-specific DEGs at 5\% false

319 discovery rate (FDR); however, csSAM identified O DEG at the same 5\% FDR level (the default

320 significance level used in csSAM).

321 Further, we performed pathway enrichment analysis with top cell type-specific genes ranked by

322 both FastMix and csSAM. Using the step-by-step csSAM, we obtained the 100 top ranked

323 genes based on csSAM estimated FDR. For fair comparison, we also extracted the top 100

324 FastMix cell type-specific DEGs, and fed both FastMix and csSAM top 100 genes to the 
medRxiv preprint doi: https://doi.org/10.1101/2021.09.14.21263182; this version posted September 27, 2021. The copyright holder for this preprint (which was not certified by peer review) is the author/funder, who has granted medRxiv a license to display the preprint in perpetuity.

It is made available under a CC-BY-NC-ND 4.0 International license .

325 ReactomePA [43] R package for pathway enrichment analysis. FastMix identified 45, 8, and 1

326 significant cell type-specific pathways for the neutrophils, non-neutrophils, and rest population,

327 respectively (Supplementary Table S1-S3). Figure 4a showed the enriched pathways identified

328 by the top 100 FastMix neutrophil-specific DEGs for high responders. The interferon (IFN)

329 immune signaling pathways were substantially presented in the high responder group,

330 including Interferon Signaling, Interferon alpha/beta signaling, Antiviral mechanism by IFN-

331 stimulated genes, Interferon gamma signaling (the top 4). In comparison, using the top 100

332 csSAM gene list for each cell type, no enriched pathway was identified for the neutrophil

333 population, only one pathway - neutrophil degranulation - was identified for the non-

334 neutrophil population, and five pathways for the rest population (Supplementary Table S4).

335 Besides the significant pathways, we also extracted the unique genes that contributed to the

336 enriched pathways from the top 100 FastMix neutrophil-specific DEG list with respect to

337 response group (Figure 4b). In particular, we identified BST2 (Tetherin/CD317), which is a key

338 host cell defense molecule in response to stimuli from IFN pathway [44, 45]. Traditional

339 understanding of BST2 expression is with mature B cells and plasmacytoid dendritic cells while

340 it has cell type-dependent variation [46]. Our analysis showed that BST2 was also expressed in

341 neutrophils, whose increased expression level (estimated linear coefficient = 1.016; please see

342 Methods section for coefficient estimation) was correlated with the high anti-HB levels after

343 Dose 3 of Engerix B.

344 Inferring cell type-specific temporal pattern from longitudinal data using FastMix 
medRxiv preprint doi: https://doi.org/10.1101/2021.09.14.21263182; this version posted September 27, 2021. The copyright holder for this preprint (which was not certified by peer review) is the author/funder, who has granted medRxiv a license to display the preprint in perpetuity.

It is made available under a CC-BY-NC-ND 4.0 International license .

345 The NIH-funded ImmPort [47] Shared Data portal (www.immport.org/shared/home) shares

346 various immunology studies with the research community. For systems immunology, the

347 activation of immune cell response is a dynamic process; therefore, longitudinal data are

348 commonly collected to investigate how the immune system responds to a certain vaccine or

349 treatment at multiple time points. It is particularly challenging to systematically integrate multi-

350 omics data over a set of time points. We downloaded SDY180 from ImmPort, which employes

351 the systems immunology approaches to investigate immune responses to Influenza (Fluzone ${ }^{\circledast}$

352 2009-2010 seasonal influenza vaccine) and Pneumococcal (Pneumovax23 ${ }^{\circledR} 23-$ valent

353 pneumococcal vaccine) vaccines [48]. For the Influenza arm, we identified 102 samples that

354 have paired flow cytometry and microarray gene expression data for 12 subjects over 8 or 9

355 time points; for the Pneumococcal arm, we have 100 samples of 12 subjects over 8 or 9 time

356 points (Supplementary Table S5). The subjects' age range from 20-50 years old; and the nine

357 time points span from 7 days before vaccination to 28 days after vaccination. In the weighted

358 FastMix model that we fitted, we included both age and time point as model covariates.

359 Following the DAFi gating hierarchy (see the Methods section), we identified lymphocytes,

360 granulocytes, monocytes, and rest population from multiple flow cytometry panels for SDY180

361 (Figure 5a and Supplementary Figure S2). The temporal pattern of the cell proportion changed

362 over time for the Influenza arm are shown in Figure $5 \mathrm{~b}$. With only the flow cytometry data, we

363 noticed that the proportion of lymphocytes had a substantial drop on Day 1 after vaccination

364 and was recovered by Day 3. 
medRxiv preprint doi: https://doi.org/10.1101/2021.09.14.21263182; this version posted September 27, 2021. The copyright holder for this preprint (which was not certified by peer review) is the author/funder, who has granted medRxiv a license to display the preprint in perpetuity.

It is made available under a CC-BY-NC-ND 4.0 International license .

365 Using a simple pre-post (i.e. between two days) comparison, the original SDY180 study [48]

366 curated an interferon module, namely M1.2, that includes genes showing significant global

367 changes in blood transcript abundance between the baseline Day 0 and Influenza Vaccine Day 1.

368 In bulk level, representative genes (CXCL10, IFIT1, and LAMP3) in the M1.2 module showed

369 consistently a peak in gene expression on Day 1 after vaccination (Figure $5 c$ and Supplementary

370 Figure S3), confirming the global finding; the temporal plots also show that the bulk expression

371 of these genes fell back to around the baseline level on and after Day 3.

372 Applying FastMix to the Influenza arm, we could further designate the specific cell population

373 that are associated with the temporal activation of these interferon genes. Among the 24 gene

374 in M1.2 module, FastMix identified 22 genes with highly significant $p$-values $(<0.05)$ for

375 lymphocyte-specific differential expression (Figure $5 d$ and Supplementary Table S6); the top 9

376 M1.2 genes ranked in the top 1\% (out of 10732 genes) of the lymphocyte-specific DE list.

377 However, the majority of the M1.2 genes showed no significance in granulocytes and

378 monocytes (Supplementary Table S6). These results strongly indicate that the activation of the

379 interferon module is lymphocyte-specific: the differential expression of interferon signaling

380 genes are driven by the up-regulation of the lymphocyte-specific expression. Though the

381 proportion of lymphocytes decreased on Day 1 after vaccination (Figure 4b), the bulk gene

382 expression of M1.2 genes increased on Day 1 (Figure 4c). The FastMix statistical inference

383 precisely linked the temporal changes in Figure $4 \mathrm{~b}$ for lymphocytes and Figure $4 \mathrm{c}$ for those

384 interferon-stimulated genes. Furthermore, FastMix produced positive estimated coefficients

385 for lymphocytes for all M1.2 genes (Supplementary Table S7), confirming the up-regulation of

386 the cell type-specific gene expression. 
387 We also looked at the cell type and age interaction terms. The lymphocyte-specific $p$-values

388 w.r.t. age for the M1.2 interferon module genes showed very strong significance (23 out of 24

389 significant $p$-values) (Figure 4e and Supplementary Table S8), whose coefficient estimates

390 showed negative association between the subject age and lymphocyte-specific expression

391 (Supplementary Table S7 and Supplementary Figure S4).

392 For completeness of method comparison, a plausible csSAM analysis would be only to compare

393 the pre- and post-vaccination groups for cell type-specific DEGs due to technical limitations.

394 Even for the simple two-group test, the csSAM approach is suboptimal, because there is no 395 appropriate way to handle the within-subject correlation structure in the multiple time points.

396 Therefore, no significant cell type-specific DEGs w.r.t. the pre- and post-vaccination groups

397 were identified at the 5\% FDR level. Using the top 100 csSAM gene list for each cell type, no 398 enriched pathway was identified for any cell type, suggesting that csSAM is inadequate for 399 performing cell type-specific DE analysis with complex study design.

400 Lastly, applying FastMix to the Pneumococcal arm, only one significant $p$-value was obtained

401 in Figure 4c-d (Supplementary Table S6 and S8). Clearly, the lymphocyte-specific interferon

402 activation was only observed in the Influenza arm, but not the Pneumococcal arm, agreeing

403 with the existing knowledge in PBMC samples [48]. The Pneumococcal arm may serve as the

404 "true negative" for our method validation; and the non-significant results showed the

405 "specificity" of the FastMix method.

406 Discriminant Analysis after FastMix 
medRxiv preprint doi: https://doi.org/10.1101/2021.09.14.21263182; this version posted September 27, 2021. The copyright holder for this preprint (which was not certified by peer review) is the author/funder, who has granted medRxiv a license to display the preprint in perpetuity.

It is made available under a CC-BY-NC-ND 4.0 International license.

407 Because FastMix is designed to take multiple types of input variables including clinical

408 parameters, it can be used to identify the relationship between the independent (e.g., subject

409 demographics) and dependent variables (e.g., response to a vaccination). For example, in our

410 experiment using the HBV vaccination data in the HVP01 study, when the subjects were

411 grouped into responding and non-responding, FastMix could calculate four scores for

412 discriminating purpose: (a) single_score, an 1-dimensional score based on all input genes; (b)

413 single_sparse_score, a 1-dimensional score based on genes with significant interactions with

414 the response; (c) multi_score, an n-dimensional score based on all genes; and (d)

415 multi_sparse_score, a multivariate score based on genes with significant interactions with the

416 response (see Supplementary Material, Section Discriminant Analysis after FastMix, for

417 technical details). Figure 4c-d showed that the discriminative scores (for straightforward

418 illustration, single_sparse_score was used in the Figure) can be plotted to identify whether age

419 is an informative factor in the discriminative analysis (i.e., classification of responding vs non-

420 responding subjects). We can clearly see the significant (Wilcoxon $p$-value $=1.9 e-16$ ) difference

421 between the responding (yellow) and non-responding (grey) groups when age was included in

422 the analysis (Figure 4c), while the difference was unclear without the age (Figure 4d). This tells

423 us that age is an important variable that is highly relevant in host immune response to the HBV

424 vaccine.

\section{Discussion}

426 Using extensive simulation studies, we showed that: (i) regression coefficients estimated by

427 FastMix had comparable mean squared errors (MSE) as those computed from Ime4 - the 
medRxiv preprint doi: https://doi.org/10.1101/2021.09.14.21263182; this version posted September 27, 2021. The copyright holder for this preprint (which was not certified by peer review) is the author/funder, who has granted medRxiv a license to display the preprint in perpetuity.

It is made available under a CC-BY-NC-ND 4.0 International license .

428 reference implementation of LMER model based on EM algorithm, (ii) depending on settings,

429 FastMix was at least 25 times, and in some cases more than 300 times faster than Ime4, and

430 (iii) FastMix was substantially more accurate (measured by MSE) than the ordinary least

431 squares (OLS) and Ridge regression. See Table 1 for more details.

432 In addition, we compared the type-I error rate and statistical power of FastMix for cell type-

433 specific differential expression (DE) analysis with an existing pipeline, cSSAM [34], using both

434 simulations and real data. FastMix achieved slightly better statistical power with much lower

435 type-I error than cSSAM, using about 10\% of csSAM's run time (see Table 1c-e). We applied the

436 FastMix pipeline to analyze the multimodal data from two clinical studies $[37,48]$ that

437 measured host responses to three different vaccines (influenza, pneumococcal, and hepatitis B).

438 Input data included bulk gene expressions, FCM, as well as clinical covariates including vaccine

439 responding groups defined by serum antibody titers as well as time points for multiple vaccine

440 doses. A common bottleneck in evaluating multimodal data integration methods using real data

441 is the lack of ground truth. Performance assessment of many preexisting methods relies on

442 subjective interpretation of their data integration results using existing knowledge. In contrast,

443 we addressed this issue by using single cell RNA-seq (scRNA-seq) data available in one of the

444 studies as an objective gold standard. Excitingly but not surprisingly, DEGs selected by

445 FastMix overlapped significantly with those selected by the cutting-edge analysis of the

446 scRNA-seq data. On the other hand, FastMix seemed to be able to select biologically

447 important genes for neutrophils that were missed by the scRNA-seq analysis. 
medRxiv preprint doi: https://doi.org/10.1101/2021.09.14.21263182; this version posted September 27, 2021. The copyright holder for this preprint (which was not certified by peer review) is the author/funder, who has granted medRxiv a license to display the preprint in perpetuity.

It is made available under a CC-BY-NC-ND 4.0 International license .

448 The general contribution of FastMix, from a statistical perspective, is the extension from the

449 traditional pairwise linear associations between multi-omics data types into a multiple

450 regression model with both fixed and random effects (LMER) that can take multiple types of

451 inputs simultaneously and infer cell type-specific biomarkers as well as signature genes based

452 on cohorts defined by experiment variates or clinical parameters. One roadblock for realizing

453 the LMER analysis in practice is the complex and slow iterative EM algorithm for estimating the

454 regression parameters. We solved this issue by an efficient moment-based method that

455 achieved similar accuracy as EM but using only a fraction of its run time (Table 1a). Note that

456 this method includes both trimming and the corresponding bias-correction, so that the

457 estimated covariance structure (used in the LMER) is robust to outliers and practically unbiased.

458 It is important to note that the LMER construction in FastMix also addressed the collinearity

459 issue in an interpretable way, without needing a black-box non-linear transformation used in

460 many of the existing multi-omics data integration approaches. Inspired by competitive tests

461 used in gene set enrichment analyses [49-51], we designed a quasi-p-value to rank and select

462 genes with significantly larger/smaller random effects (cell-type-specific effects) than most

463 other genes. We believe this approach may be applicable in other situations.

464 FastMix provides an end-to-end solution for integrative analysis of flow cytometry (FCM)

465 data and bulk transcriptomics data. FCM and transcriptomics are commonly used in

466 immunology studies. Among the 1924 experiments in the 495 studies collected by US NIAID's

467 ImmPort database (https://immport.org/shared/home) as of June 2021, the top two assay

468 types are FCM $(706 ; 36.7 \%)$ and transcription profiling $(213 ; 11.1 \%)$. However, existing solutions

469 for integrating data from transcriptomics and FCM assays for cell type-specific immune profiling 
medRxiv preprint doi: https://doi.org/10.1101/2021.09.14.21263182; this version posted September 27, 2021. The copyright holder for this preprint (which was not certified by peer review) is the author/funder, who has granted medRxiv a license to display the preprint in perpetuity.

It is made available under a CC-BY-NC-ND 4.0 International license .

470 are suboptimal. FCM data analysis mainly relies on subjective manual gating analysis, which is

471 difficult to be integrated with other computational modules. Identification of cell type-specific

472 signature genes and DEGs relies on predefined marker genes in the transcriptomics data,

473 without utilizing the FCM data that provide canonical phenotypic definitions of the cell types.

474 We previously developed a computational method - DAFi [23] - to identify cell populations

475 from FCM data in an objective way, which produces more accurate proportions of cell

476 populations in the biological sample than the subjective manual gating analysis $[23,52]$.

477 Combining DAFi and FastMix (Figure 1d) produces a novel unbiased solution for

478 immunologists to identify cell-based biomarkers, including DEGs and cell populations with

479 significantly different abundances between cohorts, from the FCM and transcriptomics data.

480 Besides synthetic data, using scRNA-seq data provides ground truth for assessing the

481 performance of FastMix. The consistency between FastMix and scRNA-seq from our

482 experiment (Figure 3) showed that FastMix can be used to infer cell type-specific knowledge

483 from bulk transcriptomics and FCM data. However, FastMix-identified biomarker genes are also

484 complementary to results of the scRNA-seq data analysis. For example, FastMix identified the

485 neutrophil-specific genes MMP9 and RSAD2/Viperin (Figure 3c), which were not found in the

486 scRNA-seq data analysis (Figure 3a). MMP9 is a regulatory factor in neutrophil migration [53]

487 and Viperin is an important anti-viral protein induced in neutrophils [54] (Figure 3c). Also,

488 FastMix identified the IFIT gene family members (IFIT1, IFIT2, and IFIT3) that can limit the

489 HBV replication [55]. Basically, FastMix provides an in-silico alternative when scRNA-seq data

490 is unavailable or unreliable. Besides inferring the cell type-specific signature genes, FastMix

491 can produce discriminative scores of model variables, which quantify the contributions of 
medRxiv preprint doi: https://doi.org/10.1101/2021.09.14.21263182; this version posted September 27, 2021. The copyright holder for this preprint (which was not certified by peer review) is the author/funder, who has granted medRxiv a license to display the preprint in perpetuity.

It is made available under a CC-BY-NC-ND 4.0 International license .

492 model variables to the sample classification. This is a unique and novel feature that previous

493 models have not provided.

494 The main limitation of FastMix is that it does not solve the well-known problem for 495 inferring characteristics of rare cell populations from bulk assay data. When the proportion of a

496 cell population is small, its contribution to the bulk gene expressions is easily overwhelmed by

497 the abundant cell populations. Even a minor change in gene regulation of the major cell types

498 can dominate the variation of the bulk gene expressions. This challenge can potentially be

499 solved if there are replicates of the same measurement, which are unfortunately usually

500 unavailable in most biomedical studies. Ideally, scRNA-seq, bulk transcriptomics, as well as FCM,

501 when available, can be integrated together for achieving the optimal performance for

502 identifying cell-based DEGs and other biomarkers for both abundant and rare cell types in the

503 whole cell type hierarchy. The estimation can also benefit from longitudinal (and repeated)

504 measurements when they are available, which will be investigated in our future work

505 Applications of FastMix can be easily extended to include metagenomics and metabolomics

506 data. For example, a straightforward application of FastMix is to identify genetic factors

507 across species to explain variation of metabolomic profiles based on microbial community

508 composition data. FastMix allows us to do "reverse engineering" from observed metabolite

509 abundances in diverse microbiome communities to infer species-specific contributions. 


\section{$510 \quad$ Figure Legend}

511 Figure 1. FastMix schematics and analytical pipeline. (a) FastMix takes three input data

512 matrices: a bulk gene expression matrix, a matrix of cell type proportions, and a matrix of

513 clinical covariates (both continues and categorical). (b) Flow chart of key steps of FastMix.

514 (Details please refer to complementary material.) i. The FastMix model utilizes linear mixed-

515 effects regression (LMER) model and mixture distribution to construct a unified regression

516 model for the three data inputs. ii. Reparametrize the FastMix model by vectorization and

517 Kronecker product so the data can be analyzed in a unified LMER model. iii. The FastMix

518 algorithm gains computational efficiency through using a novel moment-based estimator of the

519 covariance matrix $\hat{B}^{(0)}$, followed by solving for the fixed effects estimate $\widehat{\boldsymbol{\beta}}^{(1)}$ and the random

520 effects estimate $\widehat{\gamma}_{i}^{(1)}$, both of which depend on $\widehat{B}^{(0)}$. iv. In FastMix, DEG identification is

521 viewed as an outlier detection problem. It uses a trimming technique to improve the robustness

522 due to the existence of DEGs (outliers). v. After trimming, re-estimate the variance-covariance

523 matrix using the robust estimator $\widehat{B}_{\mathrm{T}}$ with bias correction, followed by re-estimating $\widehat{\boldsymbol{\beta}}$ and $\widehat{\boldsymbol{\gamma}}_{i}$

524 using $\hat{B}_{\mathrm{T}}$. vi. FastMix performs hypothesis test and constructs quasi-p-values that indicate the

525 significance of cell type-specific DEGs. (c) Using trimming improves the estimation of the

526 covariance matrix. Axes are random effect signals of two cell populations (Cell1 and Cell2); dots

527 (grey and black) are simulated data of 5000 genes, among which, 250 genes are true DEGs in

528 the Cell1 direction (black dots). Three ellipses are the density contour curves that represent the

$52995 \%$ confidence region of the centered data distribution with covariance matrices of: $B$ that is

530 the true covariance matrix shown in black, $\hat{B}^{(0)}$ that is the initial non-robust covariance 
531 estimator shown in red, and $\hat{B}_{T}$ that is the robust covariance estimator based on trimming

532 shown in green. Due to the existence of the true DEGs (outliers), $\hat{B}^{(0)}$ overestimated the true

533 covariance matrix. The trimming-based estimator $\hat{B}_{T}$ is very close to the true covariance matrix.

534 (d) Sample analytical pipeline for cell type-specific differential analysis between disease and

535 control groups by integrating flow cytometry data and bulk RNA-seq data using two newly

536 developed computational algorithms DAFi and FastMix.

537

538 Table 1. Simulation performance. (a) Comparison of FastMix implementations (FastMix

539 with independence assumption, i.e., FastMix_ind, and default FastMix with no

540 assumption on the covariance matrix) and Ime4 implementations (Ime4 with independence

541 assumption, i.e. Ime4_ind, and default Ime4 with no assumption on the covariance matrix) for

542 estimating $B$, the covariance matrix of random effects, in linear mixed effects regression

543 (LMER). Four simulation scenarios are considered: with or without true DEGs, and with or

544 without correlation between random effects. Mean computational time and mean MSE are

545 reported. Computational time is reported in seconds, and estimation accuracy is reported in

546 mean squared error (MES). MSE is defined as $\sum_{i=1}^{p} \sum_{j=1}^{p} 1 / p^{2}\left(\widehat{B}_{i j}-B_{i j}\right)^{2}$. Simulations are

547 repeated 200 times. (b) Comparison of FastMix with ordinary least squares (OLS) and Ridge

548 regression for regression coefficient, $\beta_{i j}$, estimation. The first row is the mean MSE (standard

549 deviation in brackets) defined as $1 /(m p) \sum_{i=1}^{m} \sum_{j=1}^{p}\left(\widehat{\beta}_{i j}-\beta_{i j}\right)^{2}$. The other rows are the mean

550 bias (standard deviation in brackets) of each fix effect coefficient estimation. Simulations are

551 repeated 200 times. All results are reported after multiplying by 100 for better readability. (c-e) 
552 Mean (standard deviation in brackets) of type-I error rate (c), statistical power (d), and

553 computational time reported in seconds (e) of csSAM and FastMix for cell type-specific DEG

554 detection, in the same simulation scheme repeated 200 times. The simulation design includes

555 independent random effects (i.e., cor $=0$ ) and correlated random effects (i.e., cor $=0.5$ ). True

556 cell type-specific DEGs are only assigned in cell1 and cell2 in the simulations. Type-I error rate

557 and statistical power are reported in percentage (\%).

558 Figure 2. FastMix and scRNA-seq results for HVP01 study. (a) DAFi gating strategy to identify

559 singlets, leukocytes, live leukocytes, CD66- CD45+ population (parent: live leukocytes), and

560 CD66+ CD45- population (parent: live leukocytes). (b) UMAP visualization of scRNA-seq cell type

561 clusters. Cells are colored by cluster labels derived by flow cytometry panels. (c) Overlapping of

562 the 851 (out of 13157 total genes) FastMix neutrophil-specific signature genes and the 2744

563 scRNA-seq neutrophil signature genes available in the bulk RNA-seq data. (d) Venn diagram of

564 the overlapping between the top 100 FastMix neutrophil signature genes and the scRNA-seq

565 neutrophil signature genes with $|\log \mathrm{FC}|>1$. The 39 common genes are shown in the text box.

566 Figure 3. Expression of neutrophil-specific signature genes in the scRNA-seq experiment. (a)

567 The 39 common signature genes identified by FastMix and scRNA-seq analysis (same in

568 Figure 2c). (b) The bottom 39 genes ranked by FastMix. (c) The top 39 genes ranked by

569 FastMix.

570 Figure 4. Pathway enrichment analysis for HVP01 study. (a) Enriched pathways identified by

571 the top 100 FastMix neutrophil-specific DEGs for high responders. (b) Unique genes from the 
572 CD45pCD66p. Response (i.e., neutrophil and high response) interaction DEG list that are

573 identified in the enriched pathways in (d).

574 Figure 5. FastMix analysis for SDY180. (a) DAFi gating strategy to identify lymphocytes,

575 granulocytes, and monocytes, CD45+ CD14- (parent: granulocytes and monocytes), CD45+

$576 \mathrm{CD} 14+$ (parent: granulocytes and monocytes), granulocytes (parent: CD45+ CD14-), and

577 monocytes (parent: CD45+ CD14+). (b) Boxplots of cell proportions (lymphocytes, granulocytes,

578 monocytes) over time in the Influenza vaccine study. (c) Boxplots of bulk expression levels of

579 interferon-stimulated genes (e.g., CXCL10, IFIT1, LAMP3) over time in the Influenza vaccine

580 study. Red box: matching temporal pattern change of lymphocytes proportion and bulk gene

581 expression. (d) Heatmap of -log10-transformed $p$-values for lymphocyte-specific differential

582 expression for the interferon module genes in both Influenza and Pneumococcal study arms. (e)

583 Heatmap of -log10-transformed $p$-values for lymphocyte-specific differential expression w.r.t.

584 age for the interferon module genes in both Influenza and Pneumococcal study arms. 


\section{Methods}

\section{Cell type-specific inference based on bulk tissue modeling}

587 In the regression model framework, composite tissue data can be modeled as

$$
Y_{j i}=\sum_{k=1}^{K} \operatorname{Cell}_{j k} \cdot b_{k i j}+\epsilon_{i j}, \quad i=1, \ldots, m, j=1, \ldots, n . \#(1)
$$

588 Specifically, $Y_{j i}$ is the observed bulk expression of the $i$ th gene and $j$ th sample; Cell $_{j k}$ is the

589 observed proportion of the $k$ th cell type (or cell population) in the $j$ th sample, and $b_{k i j}$ is the

590 cell type-specific expression level of the $i$ th gene and $j$ th sample contributed solely by the $k$ th

591 cell type, and $\epsilon_{i j}$ is the uncertainty in measuring Cell $_{j k}$ and $Y_{j i}$. Many downstream analyses are

592 focused to associate $b_{k i j}$ (cell type-specific gene expression) instead of $Y_{j i}$ (bulk gene

593 expression) to the clinical metadata, which can be modeled as

$$
b_{k i j}=\beta_{k i}+\sum_{p=1}^{P} \operatorname{Clin}_{j p} \cdot a_{i p k}+e_{k i j} \cdot \#(2)
$$

594 Here $\beta_{k i}$ is the baseline expression level of the $i$ th gene in the $k$ th cell type, $\mathrm{Clin}_{j p}$ is the $p$ th

595 clinical covariate associated with the $j$ th sample, $a_{i p k}$ quantifies the linear association between

596 the $p$ th clinical covariate and the $i$ th gene specific to the $k$ th cell type. In this context, cell type-

597 specific differential analysis can be conducted by testing the following hypotheses

$$
H_{0, i p k}: a_{i p k}=0, \text { v.s. } H_{1, i p k}: a_{i p k} \neq 0 . \#(3)
$$


598 For the $k$ th cell type, the $i$ th gene is a cell type-specific DEG with respect to the $p$ th clinical

599 covariate if the $p$-value from the Equation (3) hypothesis test is statistically significant.

600 Based on the above framework, one straightforward approach to perform cell type-specific

601 analysis would consist of two stages: (i) apply in silico algorithm, such as deconvolution, to

602 estimate $\hat{b}_{k i j}$; and (ii) apply a suitable DE analysis to associate $\hat{b}_{k i j}$ with the clinical data.

603 However, there is a major challenge of this approach, i.e., Equation (1) is a typical "large $p$,

604 small $n$ " problem because there are approximately Knm unknown parameters $\left(b_{k i j}\right)$ to be

605 estimated from only $\mathrm{nm}$ observations $\left(Y_{j i}\right)$. While many computational methods such as

606 nonnegative matrix factorization [56-59], regularization [60], and Bayesian methods [61-64],

607 are used to obtain approximate solutions an under-determined system for deconvolution [65],

608 the bias and variance of the estimated $\hat{b}_{k i j}$ are inevitably large, which will consequently impact

609 the accuracy of the downstream DE analysis.

610 FastMix model

611 We propose to jointly model the two-stage analysis in one unified regression model by

612 combining Equation (1) and Equation (2)

$$
\begin{aligned}
Y_{j i} & =\sum_{k=1}^{K} \operatorname{Cell}_{j k} \cdot\left(\beta_{k i}+\sum_{p=1}^{P} \operatorname{Clin}_{j p} \cdot a_{i p k}+e_{k i j}\right)+\epsilon_{i j} \\
& =\sum_{k=1}^{K} \operatorname{Cell}_{j k} \beta_{k i}+\sum_{k=1}^{K} \sum_{p=1}^{P} \operatorname{Cell}_{j k} \operatorname{Clin}_{j p} \cdot a_{i p k}+\tilde{\epsilon}_{i j} .
\end{aligned}
$$


613 Here $\tilde{\epsilon}_{i j}$ is the combined error term, $\tilde{\epsilon}_{i j}=\epsilon_{i j}+\sum_{k=1}^{K} \operatorname{Cell}_{j k} e_{k i j}, a_{i p k}$ that quantifies the

614 interaction between the $k$ th cell type and the $p$ th clinical covariate. To model the direct

615 association between the bulk gene expression and clinical covariates, we further add a main

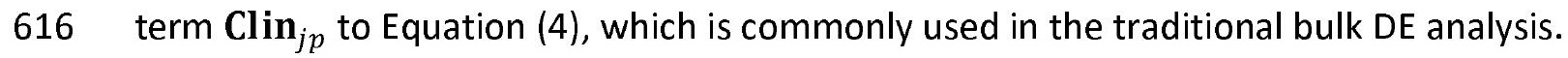

617 Therefore, the unified model includes main terms $\mathrm{Cell}_{j k}$ and $\mathbf{C l i n}_{j p}$, and their interaction term

$618 \mathrm{Cell}_{j k} \mathrm{Clin}_{j p}$, which can be stated in the standard multivariate linear regression model

$619 Y=X W+E$, or explicitly

$$
Y_{j i}=\sum_{l=1}^{L} X_{j l} \beta_{l i}+\epsilon_{i j}
$$

620 Here $X_{j l}$ is an element in matrix $X:=($ Cell Clin Cell $\times$ Clin $)$, which has $n$ rows and

$621 L=K+P+K P$ columns. We call each column of matrix $X$ a linear predictor, and $\beta_{l i}$ 's the

622 linear coefficients. Model (5) is a combination of both bulk and cell type-specific DE analyses.

623 This unified modeling approach allows us to bypass the error-prone and computationally

624 intensive parameter estimation stage (Equation (1)), and only focus on the more biological

625 interpretable DE stage for the associations between the three types of variables (cell types,

626 clinical covariates, and their interactions) with the bulk/cell type-level gene expression.

627 One important advantage of the unified model approach is that with reasonably large sample

628 size $(n>L)$, Model (5) no longer has the "large $p$, small $n$ " problem because there are only $m L$

629 unknown parameters $\left(\beta_{l i}\right)$ to be estimated with $n m$ observations $\left(Y_{j i}\right)$. FastMix does not

630 explicitly estimate the deconvoluted cell type-specific expression values; rather, it uses joint

631 modeling and techniques to be introduced in the following sections to implement a fast- 
medRxiv preprint doi: https://doi.org/10.1101/2021.09.14.21263182; this version posted September 27, 2021. The copyright holder for this preprint (which was not certified by peer review) is the author/funder, who has granted medRxiv a license to display the preprint in perpetuity.

It is made available under a CC-BY-NC-ND 4.0 International license .

632 algorithmic solution for the large-scale unified model (Equation (5)) for bulk and cell type-

633 specific DE analyses.

634 Common strategies to solve a large-scale model such as Equation (5) is to apply regularizations,

635 a.k.a. penalized regressions such as ridge [13], LASSO [14], and elastic-net [15], to increase the

636 stability and prediction accuracy of the original regression model. However, these techniques

637 have two drawbacks: (i) they shrink the estimated linear coefficients toward zero and create

638 nontrivial bias; and (ii) the best penalty parameter(s) are typically trained by time-consuming

639 cross-validation (CV) procedures, which may not always be computationally feasible for high-

640 throughput data analysis. As an alternative, we propose to use linear mixed effects regression

641 (LMER) to reduce model complexity. Specifically, we assume a two-component decomposition

642 of the unknown linear coefficient such that

$$
\beta_{l i}=\beta_{l}+\gamma_{l i}, \#(6)
$$

643 where $\beta_{l}$ is the fixed effect of the $l$ th linear predictor to the entire transcriptome, and $\gamma_{l i}$ is the

644 gene-specific random effect associated with the lth linear predictor. By combining Equations (5)

645 and (6), the FastMix model with mixed effects is

$$
Y_{j i}=\sum_{l=1}^{L} X_{j l}\left(\beta_{l}+\gamma_{l i}\right)+\epsilon_{i j}
$$

646 Using the LMER model fitting approach does not need to train hyperparameters with intensive

647 CV procedures. Also, the LMER model can shrink the estimated gene-specific linear coefficients 
medRxiv preprint doi: https://doi.org/10.1101/2021.09.14.21263182; this version posted September 27, 2021. The copyright holder for this preprint (which was not certified by peer review) is the author/funder, who has granted medRxiv a license to display the preprint in perpetuity.

It is made available under a CC-BY-NC-ND 4.0 International license .

648 toward the fixed effects (i.e. average of the entire transcriptome) instead of zero [20], thereby

649 achieving comparable variance-reduction effects with less bias.

650 The next step is to model DEGs and non-DEGs (NDEGs) based on Equation (7). In most practical

651 cases, the majority of the genes are NDEGs; and only a small fraction of the genes are truly

652 DEGs that may be used as biomarkers for specific biological conditions. In this regard, we

653 propose to approach the DEG identification problem as an outlier detection problem in more

654 general setting. Specifically, we propose to model the gene-specific random effects, $\gamma_{l i}$ 's, using

655 a mixture distribution and adapt a nonparametric empirical Bayes method [24, 25] to conduct

656 per-gene statistical inference.

657 Let $\iota$ be a binary indicator for $\operatorname{DEG}(\iota=1)$ and NDEG $(\iota=0)$. The prior probability of a gene

658 being NDEG or DEG is $P(\iota=0)=\pi_{0}$ or $P(\iota=1)=1-\pi_{0}$, respectively. The mixture

659 distribution of the multivariate vector $\gamma_{i}=\left(\gamma_{l i}, l=1, \cdots, L\right)^{\prime}$ is

$$
\gamma_{i} \sim f(\mathbf{x}), \quad f(\mathbf{x})=\pi_{0} f_{0}(\mathbf{x})+\left(1-\pi_{0}\right) f_{1}(\mathbf{x}), \#(8)
$$

660 where $\mathbf{x}$ is a dummy variable, $f_{0}(\cdot)$ is the component distribution for NDEGs and $f_{1}(\cdot)$ is the

661 component distribution for DEGs. Furthermore, it is reasonable to assume that: (i) $\pi_{0} \gg 1-\pi_{0}$,

662 i.e. most of the genes are NDEGs; (ii) the conditional distribution of the multivariate vector $\boldsymbol{\gamma}_{i}$

663 given $\iota=0$ is a $L$-dimensional normal random vector centered at the origin with covariance

664 matrix $B$; and (iii) let $D_{\alpha} \subset \mathbb{R}^{L}$ be the confidence region of $f_{0}(\cdot)$ centered at the origin with

665 probability $1-\alpha$ with a relatively large $\alpha$, then

$$
P\left(\gamma_{i} \in D_{\alpha} \mid \iota=1\right) \ll P\left(\gamma_{i} \in D_{\alpha} \mid \iota=0\right) . \#(9)
$$


666 Intuitively, Equation (9) implies that compared with NDEGs, the DEGs can be viewed as "outliers"

667 (Figure 1c). No parametric assumptions are applied to $f_{1}(\cdot)$. From the above assumptions, the

668 marginal distribution for the nonparametric empirical Bayes method is

$$
f(\mathbf{x} \mid \iota)=\left\{\begin{array}{ll}
f_{0}(\mathbf{x}):=\phi(\mathbf{x} \mid \mathbf{0}, B), & \iota=0 \\
f_{1}(\mathbf{x}), & \iota=1
\end{array} \#(10)\right.
$$

669 where $\phi(\cdot \mid \mathbf{0}, B)$ is the density function of a multivariate normal random vector defined on $\mathbb{R}^{L}$

670 with zero mean and covariance matrix $B$.

671 In summary, Equations (7), (8) and (10) specify the full FastMix model of the unified pipeline

672 for cell type-specific DE analysis:

673

$$
Y_{j i}=\sum_{l=1}^{L} X_{j l}\left(\beta_{l}+\gamma_{l i}\right)+\epsilon_{i j}, \epsilon_{i j} \sim N\left(0, \sigma_{\epsilon}^{2}\right) \text { for the LMER model; }
$$

$$
\gamma_{i} \sim f(\mathbf{x}), f(\mathbf{x})=\pi_{0} f_{0}(\mathbf{x})+\left(1-\pi_{0}\right) f_{1}(\mathbf{x}) \text { for mixture distribution; and }
$$

675

$$
f(\mathbf{x} \mid \iota)=\left\{\begin{array}{ll}
f_{0}(\mathbf{x}):=\phi(\mathbf{x} \mid \mathbf{0}, B), & \iota=0 \\
f_{1}(\mathbf{x}), & \iota=1
\end{array}\right. \text { for nonparametric empirical Bayes. }
$$

677 The FastMix model has many theoretical advantages by using a LMER model; however, fitting

678 such a large LMER model with high-throughput data is still computationally challenging. To

679 reduce the high computational cost of fitting large LMER models by conventional methods,

680 such as the iterative expectation-maximization (EM) algorithm [22], we design a highly efficient 
medRxiv preprint doi: https://doi.org/10.1101/2021.09.14.21263182; this version posted September 27, 2021. The copyright holder for this preprint (which was not certified by peer review) is the author/funder, who has granted medRxiv a license to display the preprint in perpetuity.

It is made available under a CC-BY-NC-ND 4.0 International license .

681 algorithm with a novel robust moment-based covariance estimator, which avoids the iterations

682 and convergence process, thus largely saves the computational time.

683 In the following subsections, high-level descriptions of the key steps are provided here. All

684 technical details, including the derivations, proofs, and step-by-step procedures are provided in

685 Supplementary Material.

\section{Vectorization and Kronecker product}

687 The FastMix LMER model can be concisely represented in vectorization form using Kronecker

688 product [66]

$$
\begin{gathered}
\mathbf{Y}=\mathbf{X} \boldsymbol{\beta}+\mathbf{Z} \boldsymbol{\gamma}+\boldsymbol{\epsilon} \\
\mathbf{X}:=1_{m} \otimes X=\left(\begin{array}{c}
X \\
\vdots \\
X
\end{array}\right), \quad \gamma:=\left(\begin{array}{c}
\boldsymbol{\gamma}_{1} \\
\vdots \\
\boldsymbol{\gamma}_{m}
\end{array}\right), \quad \mathbf{Z}:=I_{m} \otimes X=\left(\begin{array}{ccc}
X & & \\
& \ddots & \\
& & X
\end{array}\right) . \#(10)
\end{gathered}
$$

689 Note that $\mathbf{X}$ is $N \times L$-dimensional, $\mathbf{Z}$ is $N \times m L$-dimensional, and $\boldsymbol{\gamma}$ is $m L \times 1$-dimensional,

690 where $N=m n$ is the total number of observations. In this form, $\mathbf{Y}$ is a long vector of length $N$,

691 by column-wise stacking of the bulk gene expression matrix; $\boldsymbol{\beta}$ is the long vector of linear

692 coefficients to be estimated of the same length; and $\epsilon$ is the corresponding error vector. Now, it

693 is clear that all three types of high- and low-dimensional data are neatly combined in the form

694 of a standard LMER. The vectorized notions (in bold face and non-italic) will be used in the

695 subsequent estimation derivations; it also helps to speed up the implementation of the

696 algorithm.

\section{Moment-based estimation}


698 An initial estimation of the linear coefficients $\widehat{\boldsymbol{\beta}}_{i}^{(0)}=\left(\hat{\beta}_{l i}, \mathrm{l}=1, \cdots, \mathrm{L}\right)^{\prime}$ can be obtained through

699 fitting the multivariate linear regression in Equation (5) using the ordinary least squares (OLS)

700 criterion. $\widehat{\boldsymbol{\beta}}_{i}^{(0)}$ can be considered as a crude approximation of $\boldsymbol{\gamma}_{i}$, which contains information

701 about the covariance matrix of $\boldsymbol{\gamma}$. Denote the sample covariance matrix of $\widehat{\boldsymbol{\beta}}_{i}^{(0)}$ as $\widehat{\Sigma}_{\widehat{\boldsymbol{\beta}}^{(0)}} \in M_{L \times L}$.

702 Even for NDEGs $(\iota=0), \widehat{\Sigma}_{\widehat{\boldsymbol{\beta}}^{(0)}}$ is not an unbiased estimator of $B$. Its conditional expectation can

703 be derived as follows

$$
E\left(\widehat{\Sigma}_{\widehat{\boldsymbol{\beta}}^{(0)}} \mid \iota=0\right)=B+\sigma_{\epsilon}^{2}\left(X^{\prime} X\right)^{-1}
$$

704 Based on Equation (11), and the assumption that most genes are NDEGs $(\iota=0)$, we propose

705 the following moment-based estimator for an initial estimation of $B$

$$
\widehat{B}^{(0)}:=\widehat{\Sigma}_{\widehat{\boldsymbol{\beta}}^{(0)}}-\widehat{\sigma}_{\epsilon}^{2}\left(X^{\prime} X\right)^{-1}
$$

706 Based on the initial OLS-based estimates, the standard closed-form solutions of solving LMER

707 model are used to obtain the first set of fixed effects and random effects estimates for Equation

708 (6), denoted in $\widehat{\boldsymbol{\beta}}^{(1)}$ and $\widehat{\boldsymbol{\gamma}}_{i}^{(1)}$, respectively. The weighted least squares (WLS) estimator is used

709 to compute $\widehat{\boldsymbol{\beta}}^{(1)}$; and the empirical best linear unbiased predictor (EBLUP) is used to compute

$710 \widehat{\gamma}_{i}^{(1)}$. Both estimates depend on $\widehat{B}^{(0)}$, the initial moment-base estimate of $B$.

\section{Trimming for potential DEGs}


medRxiv preprint doi: https://doi.org/10.1101/2021.09.14.21263182; this version posted September 27, 2021. The copyright holder for this preprint (which was not certified by peer review) is the author/funder, who has granted medRxiv a license to display the preprint in perpetuity.

It is made available under a CC-BY-NC-ND 4.0 International license .

712 One of the assumptions of the FastMix model is that there is a small subset of genes that are

713 DEGs. The existence of these potential DEGs may affect the accuracy of the initial estimates of

$714 B, \boldsymbol{\beta}$, and $\boldsymbol{\gamma}_{i}$ in the previous step. We designed a three-step procedure to detect and remove

715 those potential DEGs.

716 Briefly speaking, when the DEGs are present, $\widehat{\gamma}_{i}^{(1)}$ no longer follows a multivariate normal

717 distribution. A DEG for one covariate may very likely be an NDEG for another covariate; also, it

718 is possible that a subset of covariates is not associated with any DEG (we call them

719 uninformative covariates). The three-step procedure includes: (i) use a standard normality test,

720 the Shapiro-Wilk test, to separate the informative and uninformative covariates; (ii) select the

721 subset of the standardized linear coefficient estimates pertain to the informative covariates and

722 calculate the Mahalanobis distance between the sub-vector and the origin (its theoretical

723 mean), i.e., this quantity quantifies how likely a gene is an outlier among all genes. Under the

724 assumption that most genes are NDEGs, the distance metrics of all genes form approximately a

725 chi-squared distribution; and (iii) use a pre-defined trim level (denoted as $\alpha$, which is a user-

726 defined tuning parameter with default value $\alpha=0.5$ ) to select potential DEGs based on the

727 distance metric following the chi-squared distribution.

728 After trimming of the potential DEGs that break the normality assumption, the remaining genes,

729 denoted as $S_{0} \subseteq\{1, \ldots, m\}$, will be used to refine the estimation in previous steps.

\section{Re-estimation and bias correction}


731 Now, re-estimate $B$ based on the trimmed gene list $S_{0}$. However, the trimming procedure

732 inevitably introduces bias to the sample covariance matrix, because removing genes with large

733 Mahalanobis distance artificially reduces the sample covariance matrix computed from the

734 remaining genes. To correct for this bias, we consider a truncated chi-squared distribution and

735 constructed a moment-based bias-correction for $B$ as follows

$$
\widehat{B}_{T}:=\Lambda^{-1 / 2} \widetilde{\Lambda}^{1 / 2} \widehat{\Sigma}_{\widehat{\boldsymbol{\beta}}^{(0)}} \widetilde{\Lambda}^{1 / 2} \Lambda^{-1 / 2}-\widehat{\sigma}_{\epsilon}^{2}\left(X^{\prime} X\right)^{-1} . \#(13)
$$

736 For the fixed effect, we utilize the similar idea of trimming to de-bias the fix effect estimation if

737 there are any potential unbalanced DEGs (see Supplementary Material Section 2.2 for more

738 details). The final fixed effects estimate $\widehat{\boldsymbol{\beta}}$ and random effects estimate $\widehat{\boldsymbol{\gamma}}_{i}$ are re-computed

739 using $\widehat{B}_{T}$. Figure 1c illustrates the advantage of the trimming and re-estimation procedures.

\section{$740 \quad$ Hypothesis test and quasi-p-value}

741 Traditionally, DE analysis can be performed through hypothesis testing strategies on the linear

742 coefficients (such as Equation (3)). There are mature regression F- and t-tests for the fixed

743 effects in LMER models, but not for the random effects because they are considered as

744 realizations of random variables (i.e. not unknown parameters) [67]. To overcome this

745 theoretical challenge, we developed a practical $p$-value-like quantity (called "quasi-p-value")

746 through analogy, to identify genes that have significantly larger or smaller predicted random

747 effect with a given covariate. The quasi- $p$-value is defined as

$$
\widehat{p}_{l i}:=1-\Phi\left(\frac{\left|\hat{\gamma}_{l i}\right|}{\widehat{\sigma}_{\widehat{\gamma}_{l}}}\right), \#(14)
$$


748 where $\Phi(\cdot)$ is the standard normal distribution function. Note that $\widehat{p}_{l i}$ is not a "true" $p$-value

749 because $\iota$ is a random variable, not a parameter, in the LMER model; so, we cannot test

750 hypotheses $H_{0}: \iota=0$ (i.e., NDEG) versus $H_{1}: \iota=1$ (i.e., DEG) in the classical sense. In practice,

751 the quasi- $p$-value for the random effects can be used as a practical criterion to rank and select

752 genes with strong association with the lth covariate, which are the central inference output

753 from the FastMix model. The random effects results can be interpreted as the cell type

754 marker, bulk-level, and cell type-specific DE analyses as introduced in the Results section.

755 For the completeness of the model output, the hypotheses for the fixed effects are

$$
H_{0, l}: \beta_{l}=0 \text {, versus } H_{1, l}: \beta_{l} \neq 0 . \#(15)
$$

756 The test statistic is $t_{\beta_{l}}:=\frac{\widehat{\beta}_{l}^{(1)}}{\widehat{\sigma}_{\widehat{\beta}_{l}^{(1)}}}$, which follows a t-distribution with degrees of freedom

757 approximated by the Satterthwaite's method [68]. The fixed effects tests are not gene-specific;

758 instead, these results can be interpreted as whether a clinical covariate has a statistically

759 significant impact on the whole transcriptome.

\section{Weighted FastMix model}

761 So far, the FastMix model assumes independent and identically distributed (i.i.d.) samples.

762 Sometimes, a priori knowledge may be available to weigh some samples over others; or in a

763 longitudinal study, repeated measurements are not i.i.d. samples and they tend to have block

764 interchangeable covariance structure. Such information can improve the estimation accuracy of

765 regression-type models [69]; they can be easily incorporate in the weighted FastMix model 
medRxiv preprint doi: https://doi.org/10.1101/2021.09.14.21263182; this version posted September 27, 2021. The copyright holder for this preprint (which was not certified by peer review) is the author/funder, who has granted medRxiv a license to display the preprint in perpetuity.

It is made available under a CC-BY-NC-ND 4.0 International license .

766 by constructing an appropriate weighted covariance matrix. We use techniques introduced in

767 Zhang et al. [69] (getSigma () function from the PBtest R package) to estimate the

768 weighted covariance matrix if unknown. In the simplest case, if weights are known, the

769 weighted covariance matrix is a diagonal matrix with weights in the diagonal. For the weighted

770 FastMix model, a data transformation step equivalent to the weighted least squares (WLS)

771 approach is adopted with the given weighted covariance matrix before running the FastMix

772 algorithm (see Supplementary Material).

\section{Simulation details}

\section{Simulation I}

775 Simulation I is one iteration of a comprehensive simulation scheme described in Simulation II

776 with correlation $\rho=0.5$ and balanced DEG design. Cell1 and Cell2 dimensions are visualized in

777 Figure 1c.

\section{Simulation II}

779 The simulated bulk gene expression levels are associated with $L=11$ covariates: three cell

780 proportions (Cell1, Cell2, and Cell3), two clinical covariates (Severity and Sex), and six

781 interaction terms between cell proportions and clinical covariates. The simulation design is as

782 follows.

783 1. Specifications of the fixed effects $\left(\beta_{l}\right)$ and the random effects $\left(\gamma_{l i}\right)$ of NDEGs are: 
1.1. Cell1 has an overall association with all gene expressions; Cell2 and Cell3 does not. Specifically, $\beta_{1}=1.5$ and $\beta_{2}=\beta_{3}=0$. For $\operatorname{NDEGs}(\iota=0)$, the random effects are $\gamma_{1 i}$, $\gamma_{2 i}$, and $\gamma_{3 i}$, which have marginal distribution $N\left(0, \sigma_{u}^{2}\right)$ with $\sigma_{u}=1$. distribution $N\left(0, \sigma_{e}^{2}\right)$ with $\sigma_{e}=0.8$.

1.3. Only the interaction term between Cell1 and Severity has an overall impact on the whole transcriptome $\left(\beta_{6}=0.75\right)$. For NDEGs, the Cell1-specific random effect with respect to Severity, $\gamma_{6 i}$, has marginal distribution $N\left(0, \sigma_{\alpha}^{2}\right)$ with $\sigma_{\alpha}=1.2$. $l=7, \cdots, 11)$

2. $20 \%$ of all genes are true DEGs (i.e., 1000 true DEGs). Specifications of the random effects $\left(\gamma_{l i}\right)$ of DEGs $(\iota=1)$ are: $N\left(b_{1 i}, \sigma_{u}\right)$. The true differential expression size is $\left|b_{1 i}\right|=3 \times \sigma_{u}$; the signs of $b_{1 i}$ follow a Bernoulli random variable with equal probability of being positive or negative (i.e., a balanced DEG design).

801

2.2. Genes 251 - 500 are DEGs with respect to Severity, i.e., $\gamma_{4 i} \sim N\left(b_{4 i}, \sigma_{e}\right)$. The true differential expression size is $\left|b_{4 i}\right|=3 \times \sigma_{e}$ with balanced DEG design. 
2.3. Genes 501 - 750 are DEGs for the interaction term between Cell2 and Severity (a.k.a. expression sizes are $\left|b_{7 i}\right|=3 \times \sigma_{\alpha}$ with balanced DEG design. specific DEGs with respect to Sex), i.e., $\gamma_{10 i} \sim N\left(b_{10 i}, \sigma_{\alpha}\right)$. The true differential expression sizes are $\left|b_{10 i}\right|=3 \times \sigma_{\alpha}$ with balanced DEG design.

3. Consider two correlation structures of the random effects (i.e., true $B$ matrix): either all random effects are independent (i.e., the identity matrix), or all random effects share an interchangeable correlation structure with $\rho=0.5$.

812 4. The noise term is independent and identically distributed (i.i.d.) and follows $N\left(0, \sigma_{\epsilon}^{2}\right)$ with $\sigma_{\epsilon}^{2}=0.25^{2}$

\section{Simulation III}

815 Because csSAM is limited to one binary clinical covariate design, simulated data are generated

816 as follows. The simulated bulk gene expression levels are associated with $L=7$ covariates:

817 three cell proportions (Cell1, Cell2, and Cell3), one clinical covariates (Group), and three

818 interaction terms between cell proportions and the clinical covariate.

819 1. Specifications of the fixed effects $\left(\beta_{l}\right)$ and the random effects $\left(\gamma_{l i}\right)$ of NDEGs are: 
1.1. Cell1 has an overall association with all gene expressions; Cell2 and Cell3 does not. Specifically, $\beta_{1}=1.5$ and $\beta_{2}=\beta_{3}=0$. For $\operatorname{NDEGs}(\iota=0)$, the random effects are $\gamma_{1 i}$, $\gamma_{2 i}$, and $\gamma_{3 i}$, which have marginal distribution $N\left(0, \sigma_{u}^{2}\right)$ with $\sigma_{u}=1$. has marginal distribution $N\left(0, \sigma_{e}^{2}\right)$ with $\sigma_{e}=0.8$. whole transcriptome for $\beta_{5}=0.5, \beta_{6}=0.75$, and $\beta_{7}=0$, respectively. For NDEGs, the random effects $\gamma_{5 i}, \gamma_{6 i}$, and $\gamma_{7 i}$ have marginal distribution $N\left(0, \sigma_{\alpha}^{2}\right)$ with $\sigma_{\alpha}=1.2$.

2. There are 500 true DEGs. Specifications of the random effects $\left(\gamma_{l i}\right)$ of DEGs $(\iota=1)$ are: is $\left|b_{5 i}\right|=3 \times \sigma_{\alpha}$ with balanced DEG design.

3. Consider two correlation structures of the random effects (i.e., true $B$ matrix): either all DEGs with respect to Group), i.e., $\gamma_{6 i} \sim N\left(b_{6 i}, \sigma_{\alpha}\right)$. The true differential expression size is $\left|b_{6 i}\right|=3 \times \sigma_{\alpha}$ with balanced DEG design. 
medRxiv preprint doi: https://doi.org/10.1101/2021.09.14.21263182; this version posted September 27, 2021. The copyright holder for this preprint (which was not certified by peer review) is the author/funder, who has granted medRxiv a license to display the preprint in perpetuity.

It is made available under a CC-BY-NC-ND 4.0 International license .

841 The FCM dataset in the HVP01 Study (https://clinicaltrials.gov/ct2/show/NCT03083158) are

842 provided by Kollmann lab, which has 75 FCS files (15 subjects across 5 visits - Day 0, 1, 3, 7 and

843 14). The markers included in the reagent panel can be found in Supplementary Table S9. DAFi

844 [23] (https://github.com/JCVenterlnstitute/DAFi-gating) was applied to identify the neutrophil

845 cell population following a predefined gating sequence (Figure 2a): Singlets (FSC-A vs FSC-H) ->

846 Leukocytes (FSC-A vs SSC-A) -> Live Leukocytes (Viability vs SSC-A) -> Neutrophils (CD66 vs

847 CD45). Proportions of neutrophils and their 2D dot plots for all 75 FCS files can be found in

848 Supplementary File and Supplementary Figures S5-6 (in two batches May and August). A single

849 set of DAFi-gating boundaries was used to identify the natural shapes of neutrophils in each

850 batch to avoid using abrupt cutoffs in the manual gating analysis and to provide straightforward

851 cross-sample comparison.

852 The second study we analyzed is the SDY180 on ImmPort

853 (https://www.immport.org/shared/study/SDY180), which is focused on immune responses to

854 influenza and pneumococcal vaccines [48]. Among all the reagent panels used in SDY180, two

855 of them contain CD45 and CD14 for us to define the granulocytes and monocytes

856 (Supplementary Table S9). The 302 corresponding FCS files of the two panels are from 36

857 subjects across 8 visits. DAFi was applied to identify three major types of cells from the FCM

858 data (Figure 5a): Lymphocytes (FSC-A vs SSC-A), Granulocytes (CD45 vs CD14 followed by back-

859 gating on FSC-A vs SSC-A), and Monocytes (CD45 vs CD14 followed by back-gating on FSC-A vs

860 SSC-A). 
medRxiv preprint doi: https://doi.org/10.1101/2021.09.14.21263182; this version posted September 27, 2021. The copyright holder for this preprint (which was not certified by peer review) is the author/funder, who has granted medRxiv a license to display the preprint in perpetuity.

It is made available under a CC-BY-NC-ND 4.0 International license .

\section{Data Availability}

862 The HVP01 dataset (https://clinicaltrials.gov/ct2/show/NCT03083158) is a clinical study

863 conducted by University of British Columbia focused on Hepatitis B vaccine Engerix-B. This

864 study has 16 healthy subjects from two cohorts: young adults (aged 40-60) and old adults (aged

865 61-80). Both RNA-seq gene expressions and flow cytometry data are available across multiple

866 visits before and after the vaccination from the same whole blood samples. Primary outcome of

867 this study is the antibody response to the first dose of Hepatitis B vaccine.

868 The SDY180 dataset is downloaded from the ImmPort Shared Data portal

869 (http://www.immport.org). This study has 18 young and healthy adult volunteers (aged 18-64)

870 randomly assigned to three study groups ( $n=6$ subjects/group) receiving a single intramuscular

871 dose of 2009-2010 seasonal influenza (Fluzone, Sanofi Pasteur, PA), pneumococcal vaccine

872 (Pneumovax23, Merck, NJ), or placebo (saline). Blood samples were collected at multiple time

873 visits, from 7 days before vaccination to 28 days after vaccination, for microarray, whole-blood

874 flow cytometry, and serum analysis of neutralizing antibodies.

875 Data preprocessing details are in Supplementary Material.

\section{Acknowledgements}

877 This work is partially funded by NIH/NIAID UH2AI132342, the Human Vaccines Project, the

878 Respiratory Pathogens Research Center (NIAID contract number HHSN272201200005C) and the

879 University of Rochester CTSA award number UL1 TR002001 from the National Center for

880 Advancing Translational Sciences of the National Institutes of Health. The content is solely the 
medRxiv preprint doi: https://doi.org/10.1101/2021.09.14.21263182; this version posted September 27, 2021. The copyright holder for this preprint (which was not certified by peer review) is the author/funder, who has granted medRxiv a license to display the preprint in perpetuity.

It is made available under a CC-BY-NC-ND 4.0 International license .

881 responsibility of the authors and does not necessarily represent the official views of the

882 National Institutes of Health.

\section{Author Contributions}

$884 \mathrm{XQ}$ and $\mathrm{YQ}$ conceived the project. HS and XQ designed and implemented the FastMix model,

885 including simulations. TRK and RHS provided HVP data and guidance on method applications.

886 AM processed the flow cytometry data. BDA processed the scRNA-seq data. YZ led the data

887 analytical experiments and assessed the model performance. YZ and HS drafted the manuscript.

$888 Y Q$ and $X Q$ revised the manuscript. All authors read and agreed on the manuscript. 


\section{References}

890 1. Pinu, F.R., et al., Systems biology and multi-omics integration: viewpoints from the metabolomics research community. Metabolites, 2019. 9(4): p. 76.

892 2. Aevermann, B.D., et al., Machine learning-based single cell and integrative analysis reveals that baseline $m D C$ predisposition predicts protective Hepatitis $B$ vaccine response. medRxiv, 2021.

3. $\mathrm{Li}, \mathrm{Y}$., et al., Advances in bulk and single-cell multi-omics approaches for systems biology and precision medicine. Briefings in Bioinformatics, 2021.

4. Consortium, H.-I., Multicohort analysis reveals baseline transcriptional predictors of influenza vaccination responses. Science immunology, 2017. 2(14).

5. Tomic, A., et al., SIMON, an automated machine learning system, reveals immune signatures of influenza vaccine responses. The Journal of Immunology, 2019. 203(3): p. 749-759.

6. Noecker, C., et al., Metabolic model-based integration of microbiome taxonomic and metabolomic profiles elucidates mechanistic links between ecological and metabolic variation. MSystems, 2016. 1(1): p. e00013-15.

7. McCall, M.N., et al., A systems genomics approach uncovers molecular associates of RSV severity. bioRxiv, 2020.

8. Abdi, H. and L.J. Williams, Principal component analysis. Wiley interdisciplinary reviews: computational statistics, 2010. 2(4): p. 433-459.

9. Hardoon, D.R., S. Szedmak, and J. Shawe-Taylor, Canonical correlation analysis: An overview with application to learning methods. Neural computation, 2004. 16(12): p. 2639-2664.

10. Abdi, H., Partial least square regression (PLS regression). Encyclopedia for research methods for the social sciences, 2003. 6(4): p. 792-795.

11. Van der Maaten, L. and G. Hinton, Visualizing data using t-SNE. Journal of machine learning research, 2008. 9(11).

12. McInnes, L., J. Healy, and J. Melville, Umap: Uniform manifold approximation and projection for dimension reduction. arXiv preprint arXiv:1802.03426, 2018.

13. Hoerl, A.E. and R.W. Kennard, Ridge regression: Biased estimation for nonorthogonal problems. Technometrics, 1970. 12(1): p. 55-67.

14. Tibshirani, R., Regression shrinkage and selection via the lasso. Journal of the Royal Statistical Society: Series B (Methodological), 1996. 58(1): p. 267-288.

15. Zou, H. and T. Hastie, Regularization and variable selection via the elastic net. Journal of the royal statistical society: series B (statistical methodology), 2005. 67(2): p. 301-320.

16. Singh, A., et al., DIABLO: an integrative approach for identifying key molecular drivers from multi-omics assays. Bioinformatics, 2019. 35(17): p. 3055-3062.

17. Peng, C., et al., A latent unknown clustering integrating multi-omics data (LUCID) with phenotypic traits. Bioinformatics, 2020. 36(3): p. 842-850.

18. Jin, S., L. Zhang, and Q. Nie, scAl: an unsupervised approach for the integrative analysis of parallel single-cell transcriptomic and epigenomic profiles. Genome biology, 2020. 21(1): p. 1-19. 
931 19. Cao, K., et al., Unsupervised topological alignment for single-cell multi-omics integration.

932

933

934

935

936

937

938

939

940

941

942

943

944

945

946

947

948

949

950

951

952

953

954

955

956

957

958

959

960

961

962

963

964

965

966

967

968

969

970

971

972

973

Bioinformatics, 2020. 36(Supplement_1): p. i48-i56.

20. Maldonado, Y.M., Mixed models, posterior means and penalized least-squares. Lecture Notes-Monograph Series, 2009: p. 216-236.

21. Zhang, S., et al., MatchMixeR: a cross-platform normalization method for gene expression data integration. Bioinformatics, 2020. 36(8): p. 2486-2491.

22. Bates, D., et al., Fitting linear mixed-effects models using Ime4. arXiv preprint arXiv:1406.5823, 2014.

23. Lee, A.J., et al., DAFi: A directed recursive data filtering and clustering approach for improving and interpreting data clustering identification of cell populations from polychromatic flow cytometry data. Cytometry A, 2018. 93(6): p. 597-610.

24. Efron, B., et al., Empirical Bayes analysis of a microarray experiment. Journal of the American statistical association, 2001. 96(456): p. 1151-1160.

25. Qiu, X., L. Klebanov, and A. Yakovlev, Correlation between gene expression levels and limitations of the empirical Bayes methodology for finding differentially expressed genes. Statistical applications in genetics and molecular biology, 2005. 4(1).

26. Khanam, A., et al., Blockade of neutrophil's chemokine receptors CXCR1/2 abrogate liver damage in acute-on-chronic liver failure. Frontiers in immunology, 2017. 8: p. 464.

27. Le, P.-H., et al., Clinical Predictors for Neutrophil-to-Lymphocyte Ratio Changes in Patients with Chronic Hepatitis B Receiving Peginterferon Treatment. in vivo, 2017. 31(4): p. 723-729.

28. Tang, B.M., et al., Neutrophils-related host factors associated with severe disease and fatality in patients with influenza infection. Nature communications, 2019. 10(1): p. 1-13.

29. Cui, Z., et al., Super-delta2: An Enhanced Differential Expression Analysis Procedure for Multi-Group Comparisons of RNA-seq Data. Bioinformatics, 2021.

30. Liu, Y., J. Zhang, and X. Qiu, Super-delta: a new differential gene expression analysis procedure with robust data normalization. BMC Bioinformatics, 2017. 18(1): p. 582.

31. Maronna, R.A. and V.J. Yohai, The behavior of the Stahel-Donoho robust multivariate estimator. Journal of the American Statistical Association, 1995. 90(429): p. 330-341.

32. Maronna, R.A. and R.H. Zamar, Robust estimates of location and dispersion for highdimensional datasets. Technometrics, 2002. 44(4): p. 307-317.

33. Rousseeuw, P.J. and K.V. Driessen, A fast algorithm for the minimum covariance determinant estimator. Technometrics, 1999. 41(3): p. 212-223.

34. Shen-Orr, S.S., et al., Cell type-specific gene expression differences in complex tissues. Nature methods, 2010. 7(4): p. 287-289.

35. Tusher, V.G., R. Tibshirani, and G. Chu, Significance analysis of microarrays applied to the ionizing radiation response. Proceedings of the National Academy of Sciences, 2001. 98(9): p. 5116-5121.

36. Wooden, S.L. and W.C. Koff, The Human Vaccines Project: Towards a comprehensive understanding of the human immune response to immunization. Human vaccines \& immunotherapeutics, 2018. 14(9): p. 2214-2216.

37. Shannon, C.P., et al., Multi-omic data integration allows baseline immune signatures to predict hepatitis $B$ vaccine response in a small cohort. Frontiers in immunology, 2020.11. 
974

975

976

977

978

979

980

981

982

983

984

985

986

987

988

989

990

991

992

993

994

995

996

997

998

999

1000

1001

1002

1003

1004

1005

1006

1007

1008

1009

1010

1011

1012

1013

1014

1015

1016

1017

38. Picelli, S., et al., Full-length RNA-seq from single cells using Smart-seq2. Nature protocols, 2014. 9(1): p. 171-181.

39. Keating, G.M. and S. Noble, Recombinant hepatitis $B$ vaccine (Engerix- ${ }^{\circledR}$ ). Drugs, 2003. 63(10): p. 1021-1051.

40. Blondel, V.D., et al., Fast unfolding of communities in large networks. Journal of statistical mechanics: theory and experiment, 2008. 2008(10): p. P10008.

41. Kiselev, V.Y., et al., SC3: consensus clustering of single-cell RNA-seq data. Nature methods, 2017. 14(5): p. 483-486.

42. $\mathrm{Xu}, \mathrm{R}$., et al., Low expression of CXCR1/2 on neutrophils predicts poor survival in patients with hepatitis $B$ virus-related acute-on-chronic liver failure. Scientific reports, 2016. 6(1): p. 1-9.

43. $\mathrm{Yu}, \mathrm{G}$. and Q.-Y. He, ReactomePA: an R/Bioconductor package for reactome pathway analysis and visualization. Molecular BioSystems, 2016. 12(2): p. 477-479.

44. Blasius, A.L., et al., Bone marrow stromal cell antigen 2 is a specific marker of type I IFNproducing cells in the naive mouse, but a promiscuous cell surface antigen following IFN stimulation. The Journal of Immunology, 2006. 177(5): p. 3260-3265.

45. Sarojini, S., T. Theofanis, and C.S. Reiss, Interferon-induced tetherin restricts vesicular stomatitis virus release in neurons. DNA and cell biology, 2011. 30(12): p. 965-974.

46. Miyagi, E., et al., Vpu enhances HIV-1 virus release in the absence of Bst-2 cell surface down-modulation and intracellular depletion. Proceedings of the National Academy of Sciences, 2009. 106(8): p. 2868-2873.

47. Bhattacharya, S., et al., ImmPort, toward repurposing of open access immunological assay data for translational and clinical research. Scientific data, 2018. 5: p. 180015.

48. Obermoser, G., et al., Systems scale interactive exploration reveals quantitative and qualitative differences in response to influenza and pneumococcal vaccines. Immunity, 2013. 38(4): p. 831-844.

49. Gatti, D.M., et al., Heading down the wrong pathway: on the influence of correlation within gene sets. BMC genomics, 2010. 11(1): p. 1-10.

50. Wu, D. and G.K. Smyth, Camera: a competitive gene set test accounting for inter-gene correlation. Nucleic acids research, 2012. 40(17): p. e133-e133.

51. Zhang, Y., et al., FUNNEL-GSEA: FUNctioNal ELastic-net regression in time-course gene set enrichment analysis. Bioinformatics, 2017. 33(13): p. 1944-1952.

52. Burel, J.G., et al., An integrated workflow to assess technical and biological variability of cell population frequencies in human peripheral blood by flow cytometry. The Journal of Immunology, 2017. 198(4): p. 1748-1758.

53. Kolaczkowska, E., et al., Neutrophil elastase activity compensates for a genetic lack of matrix metalloproteinase - 9 (MMP - 9) in leukocyte infiltration in a model of experimental peritonitis. Journal of leukocyte biology, 2009. 85(3): p. 374-381.

54. Hinson, E.R., et al., Viperin is highly induced in neutrophils and macrophages during acute and chronic lymphocytic choriomeningitis virus infection. The Journal of Immunology, 2010. 184(10): p. 5723-5731.

55. Pei, R., et al., Interferon-induced proteins with tetratricopeptide repeats 1 and 2 are cellular factors that limit hepatitis B virus replication. Journal of innate immunity, 2014. 6(2): p. 182-191. 
1018

1019

1020

1021

1022

1023

1024

1025

1026

1027

1028

1029

1030

1031

1032

1033

1034

1035

1036

1037

1038

1039

1040

1041

1042

1043

1044

1045

1046

1047

1048

1049

1050

1051
56. Gaujoux, R. and C. Seoighe, Semi-supervised Nonnegative Matrix Factorization for gene expression deconvolution: a case study. Infection, Genetics and Evolution, 2012. 12(5): p. 913-921.

57. Venet, D., et al., Separation of samples into their constituents using gene expression data. Bioinformatics, 2001. 17(suppl_1): p. S279-S287.

58. Lähdesmäki, H., et al., In silico microdissection of microarray data from heterogeneous cell populations. BMC bioinformatics, 2005. 6(1): p. 54.

59. Repsilber, D., et al., Biomarker discovery in heterogeneous tissue samples-taking the insilico deconfounding approach. BMC bioinformatics, 2010. 11(1): p. 1-15.

60. Newman, A.M., et al., Robust enumeration of cell subsets from tissue expression profiles. Nature methods, 2015. 12(5): p. 453-457.

61. Quon, G., et al., Computational purification of individual tumor gene expression profiles leads to significant improvements in prognostic prediction. Genome medicine, 2013. 5(3): p. 29.

62. Zhang, Y., et al., The effect of tissue composition on gene co-expression. Briefings in Bioinformatics, 2019.

63. Qiao, W., et al., PERT: a method for expression deconvolution of human blood samples from varied microenvironmental and developmental conditions. PLoS Comput Biol, 2012. 8(12): p. e1002838.

64. Quon, G. and Q. Morris, ISOLATE: a computational strategy for identifying the primary origin of cancers using high-throughput sequencing. Bioinformatics, 2009. 25(21): p. 2882-2889.

65. Mohammadi, S., et al., A critical survey of deconvolution methods for separating cell types in complex tissues. Proceedings of the IEEE, 2016. 105(2): p. 340-366.

66. Horn, R.A., R.A. Horn, and C.R. Johnson, Topics in matrix analysis. 1994: Cambridge university press.

67. Robinson, G.K., That BLUP is a good thing: the estimation of random effects. Statistical science, 1991. 6(1): p. 15-32.

68. Satterthwaite, F.E., An approximate distribution of estimates of variance components. Biometrics bulletin, 1946. 2(6): p. 110-114.

69. Zhang, Y., et al., Highly efficient hypothesis testing methods for regression-type tests with correlated observations and heterogeneous variance structure. BMC Bioinformatics, 2019. 20(1): p. 185. 
Bulk gene expression matrix

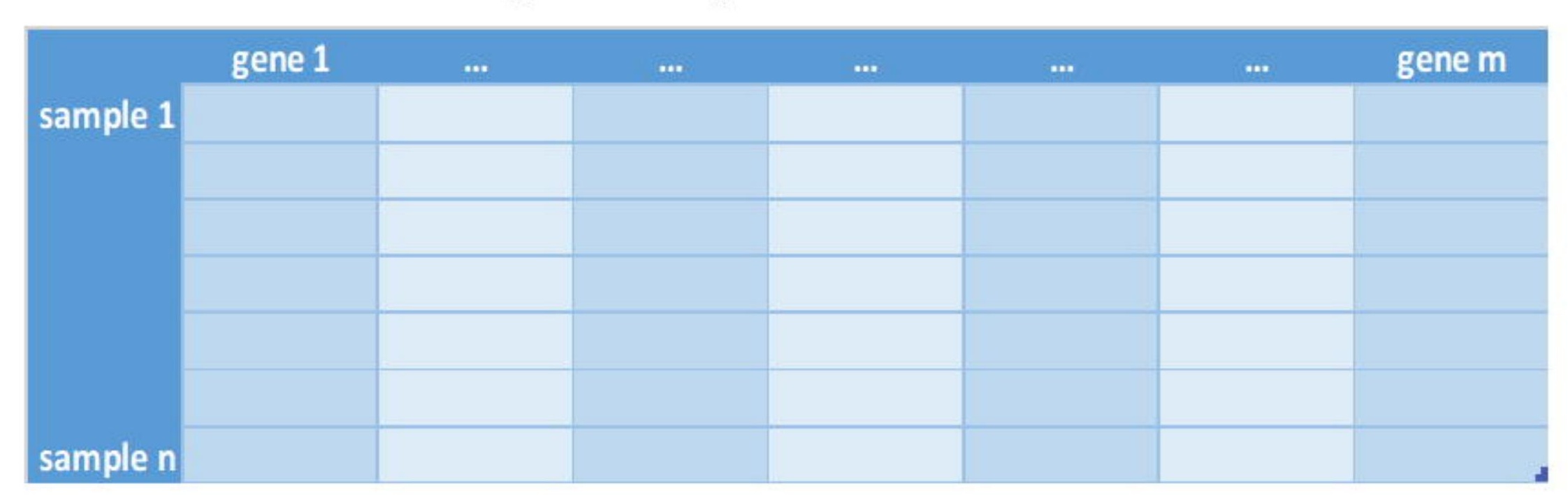

Cell type proportions

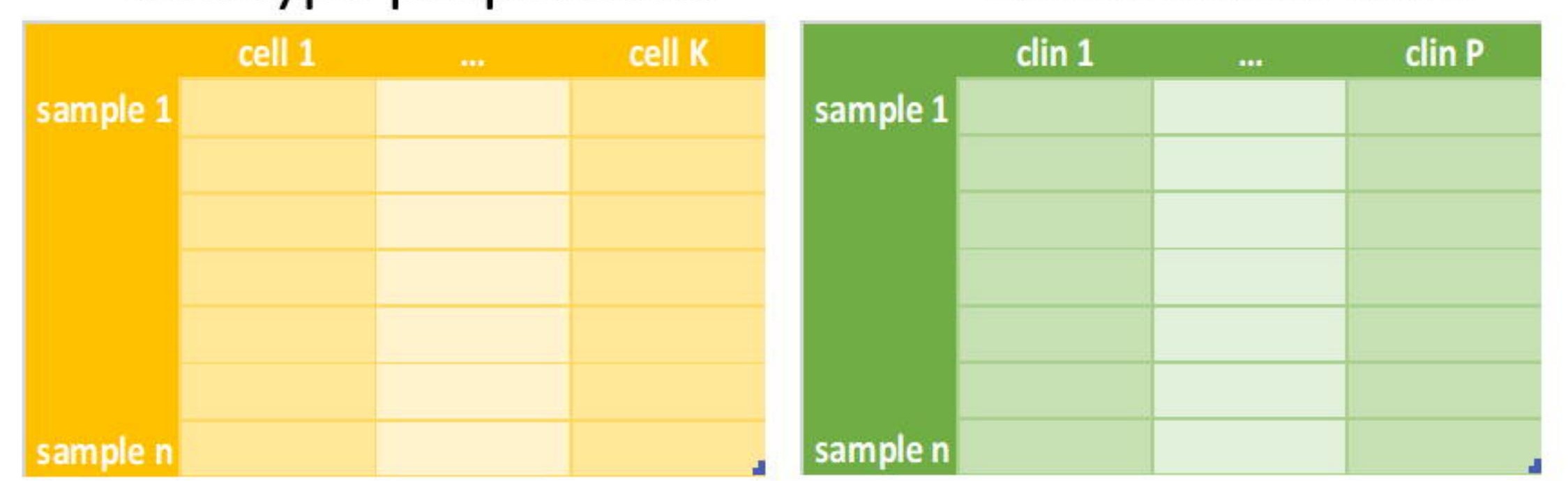

C

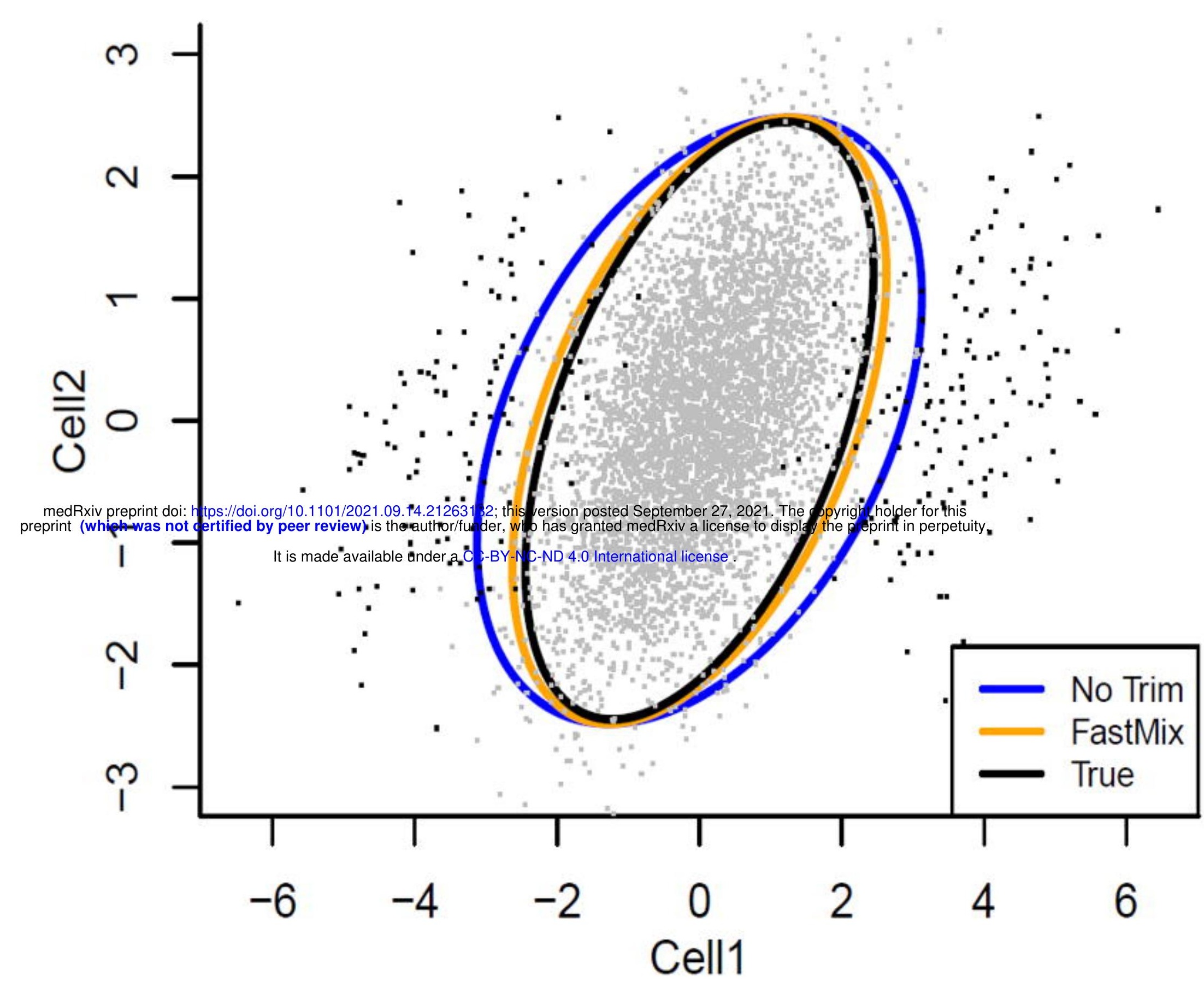

i. FastMix model

$Y_{j i}=\sum_{l=1}^{L} X_{j l}\left(\beta_{l}+\gamma_{l i}\right)+\epsilon_{i j}, \quad \gamma_{i} \sim f(\mathbf{x} \mid \iota), \quad \epsilon_{i j} \sim N\left(0, \sigma_{\epsilon}^{2}\right)$.
$f(\mathbf{x} \mid \iota)= \begin{cases}f_{0}(\mathbf{x}):=\phi(\mathbf{x} \mid \mathbf{0}, B), & \iota=0, \\ f_{1}(\mathbf{x}), & \iota=1 .\end{cases}$

ii. Vectorization and Kronecker product

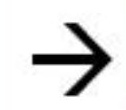

$\mathbf{Y}=\mathbf{X} \boldsymbol{\beta}+\mathbf{Z} \boldsymbol{\gamma}+\boldsymbol{\epsilon}, \quad \mathbf{X}=1_{m} \otimes X=\left(\begin{array}{c}X \\ \vdots \\ X\end{array}\right), \quad \boldsymbol{\gamma}=\left(\begin{array}{c}\gamma \cdot 1 \\ \vdots \\ \gamma \cdot m\end{array}\right)$

$\mathbf{Z}:=I_{m} \otimes X=\operatorname{diag}(X, \ldots, X)=\left(\begin{array}{lll}X & & \\ & \ddots & \\ & & X\end{array}\right)$.

$\checkmark$

iii. Moment-based estimation

$$
\widehat{B}^{(0)}, \widehat{\boldsymbol{\beta}}^{(1)}, \widehat{\boldsymbol{\gamma}}_{i}^{(1)}
$$

iv. Trimming for potential DEGs

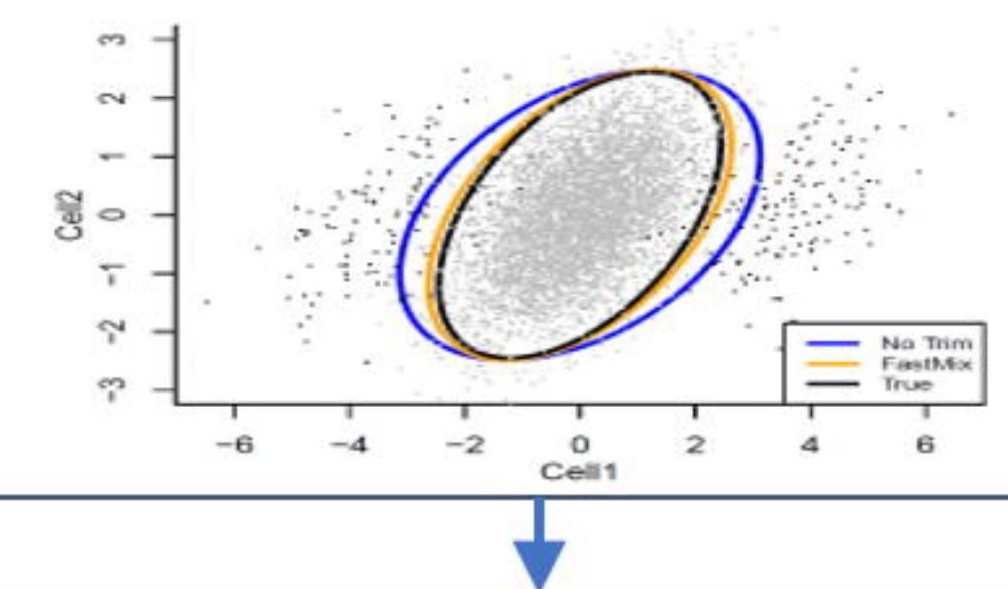

v. Re-estimation and bias correction

$$
\widehat{B}^{(0)} \rightarrow \widehat{B}_{\mathrm{T}}, \widehat{\boldsymbol{\beta}}^{(1)} \rightarrow \widehat{\boldsymbol{\beta}}, \widehat{\boldsymbol{\gamma}}_{i}^{(1)} \rightarrow \widehat{\boldsymbol{\gamma}}_{i}
$$

vi. Hypothesis test and quasi-p-value

$$
\begin{gathered}
H_{0, l}: \beta_{l}=0, \quad \text { v. } \quad H_{1, l}: \beta_{l} \neq 0 . \\
\hat{p}_{l i}:=1-\Phi\left(\frac{\left|\hat{\gamma}_{i}\right|}{\hat{\sigma}_{\hat{\gamma}_{l}}}\right)
\end{gathered}
$$

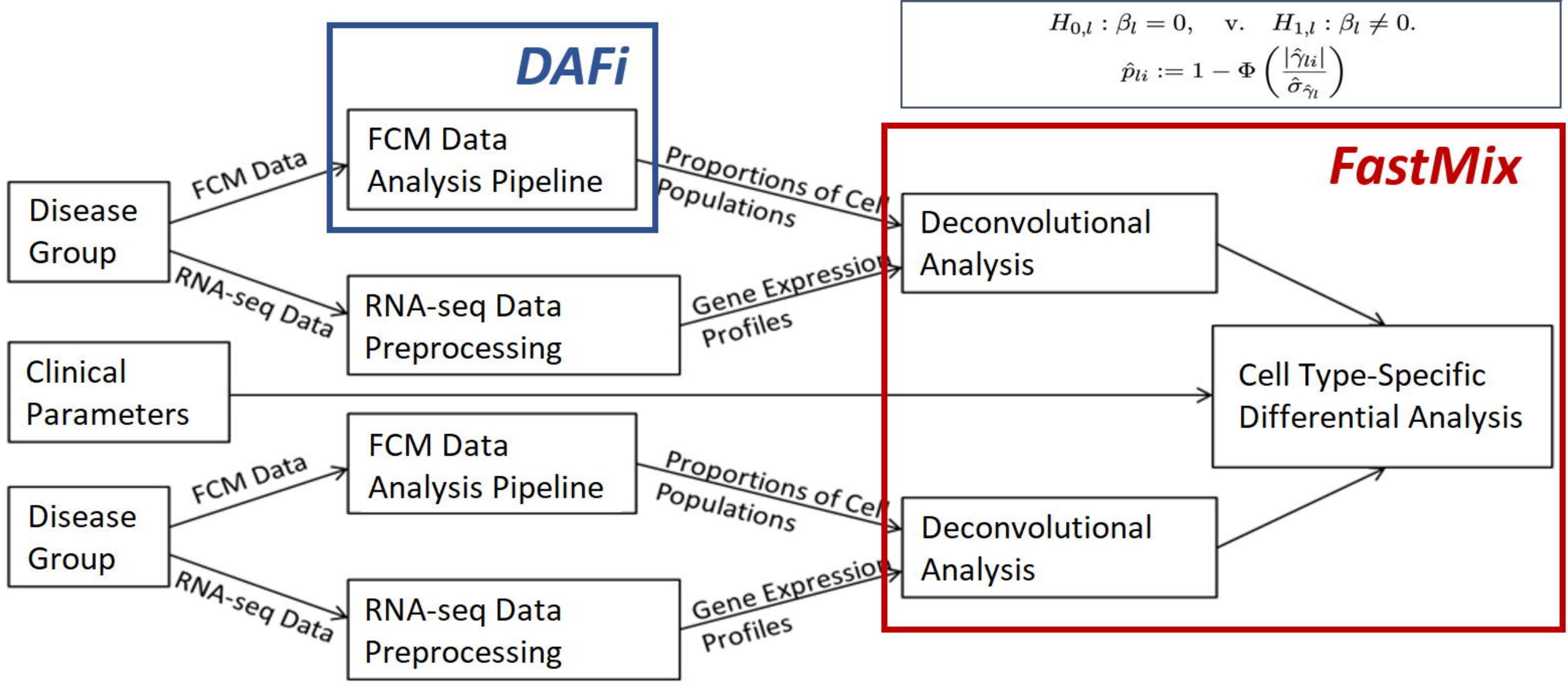




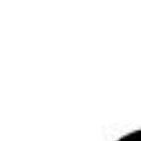
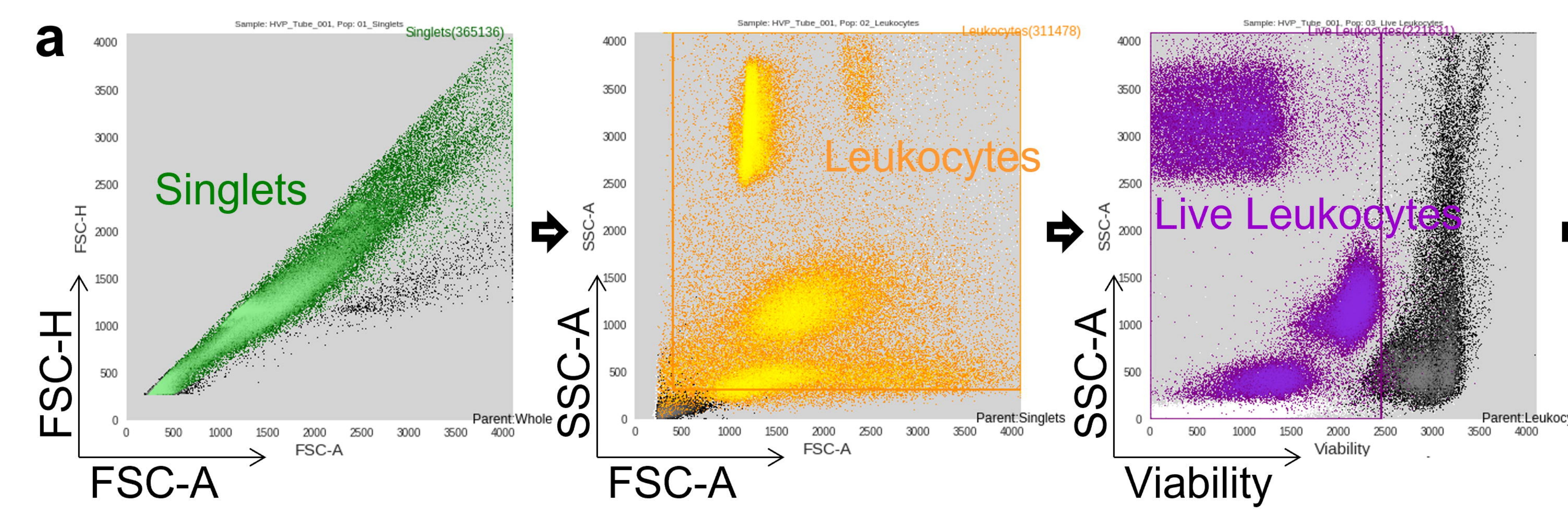

$\Rightarrow$

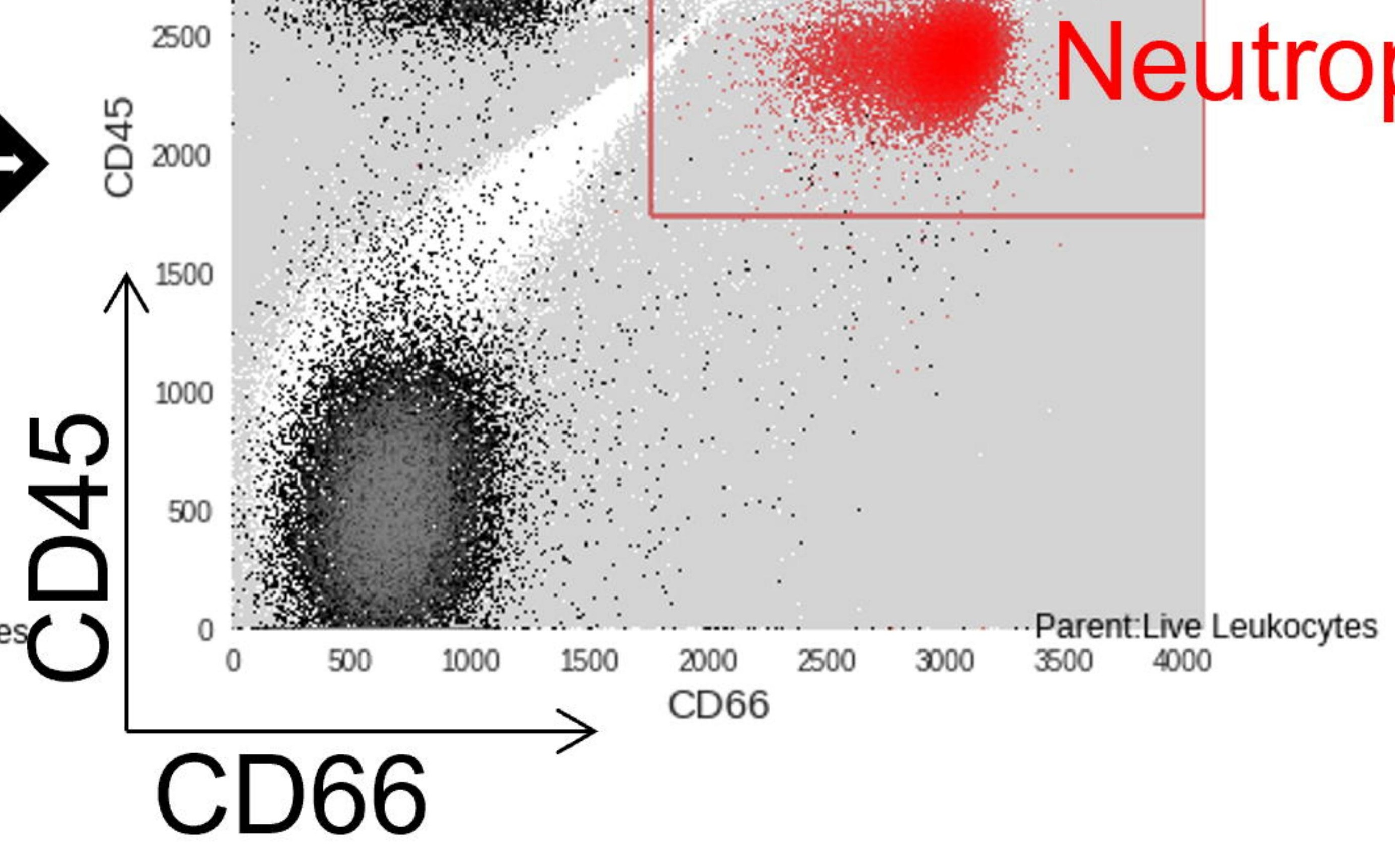

Neutrophils
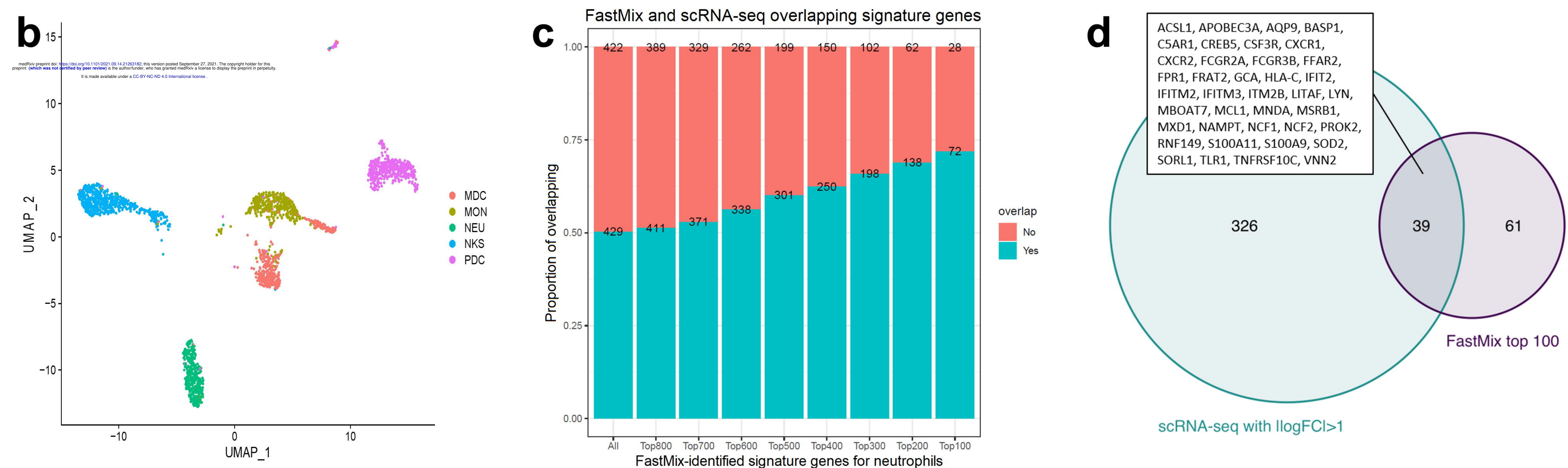

scRNA-seq with $\mid \log F C l>1$ 


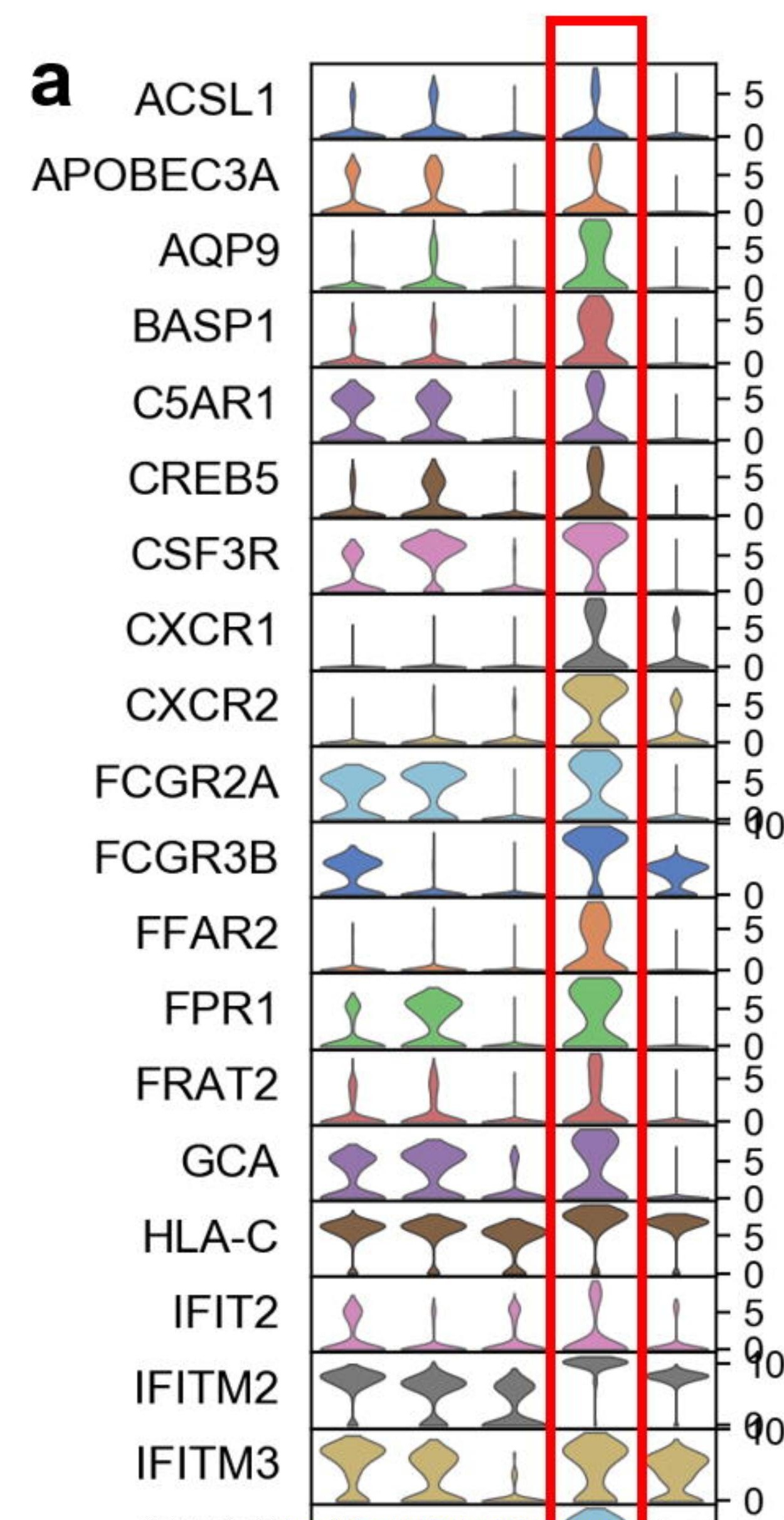

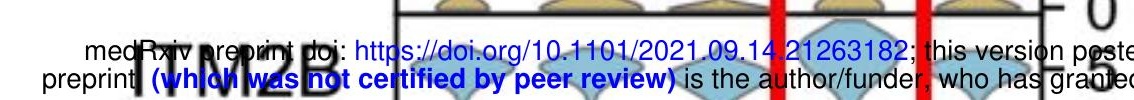

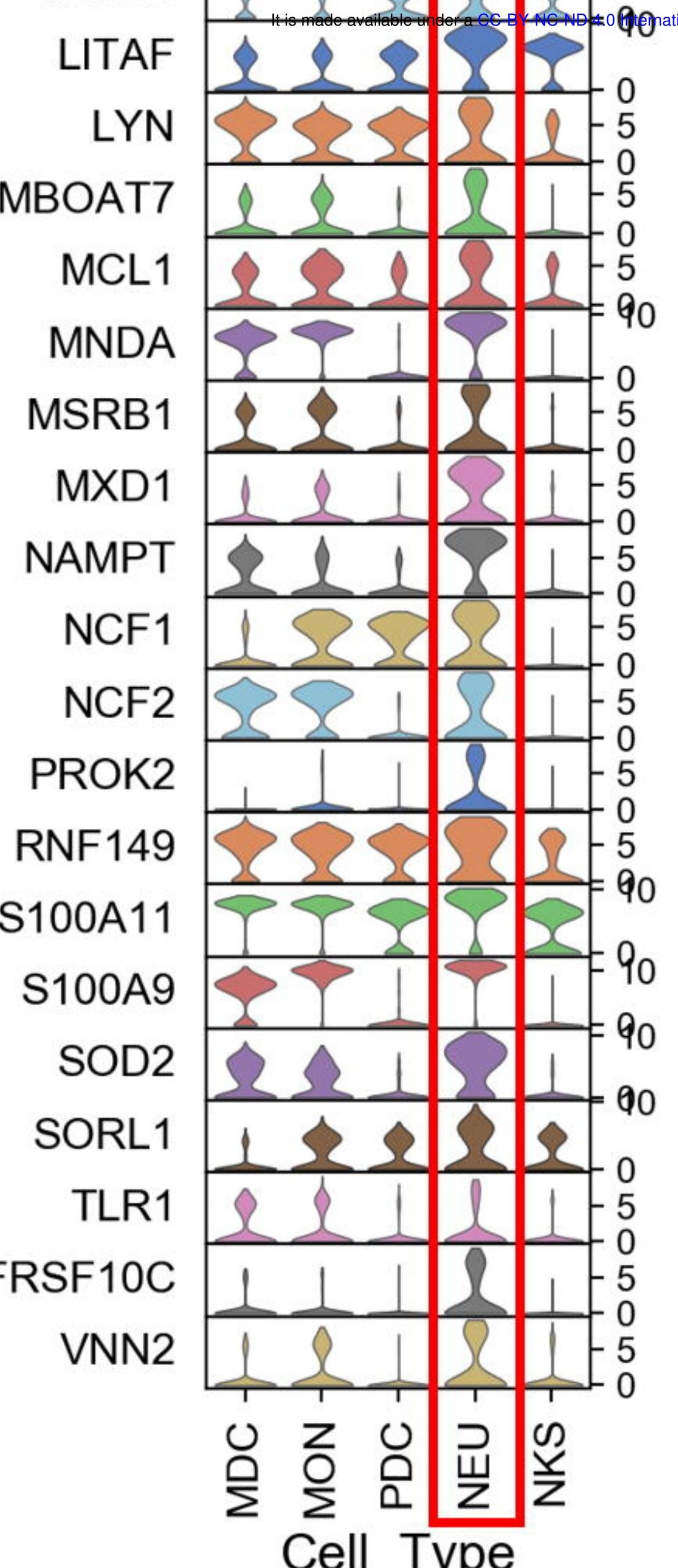

XXbac-BPG181B23.7

AC007278.2

PLCXD2

TAF11

NUP188

SGF29

PUS10

TOMM22

NFKBID

NID1

CBWD7

ZNF28

SNAPIN

TBL3

NAf PSD7

GDPD5

ABHD14B

ATF1

JUN

POLE

FOPNL

SCRN3

COQ7

ZNF639

PRR11

ATIC

ATP5D

DUS3L

EIF2B2

SRD5A1

SUGT1

RP11-449P15.2

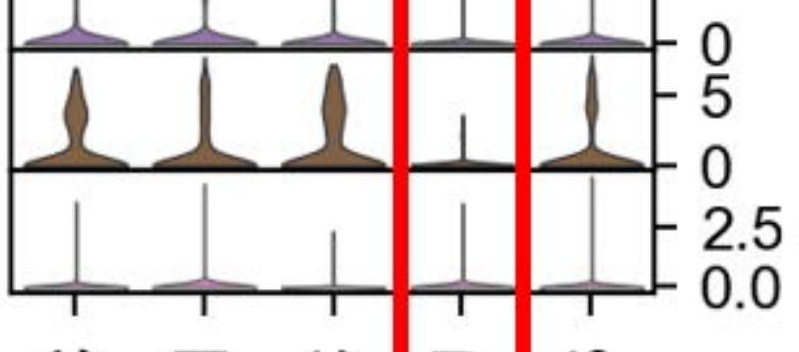

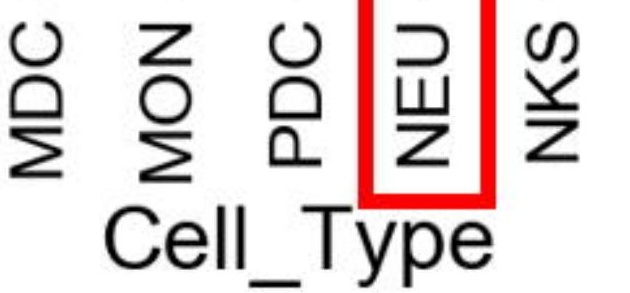

C FCGR3B

RSAD2

ACSL1

AQP9

IFITM3

BASP1

CXCR2

MME

CSF3R

IFIT2

MMP9

NAMPT

IFITM2

DYSF

CXCR1

MGAM

MNDA

MX1

KCNJ15

IFIT1

SOD2

TNFRSF10C

PROK2

IFIT3

SELL

FPR1

IL1RN

FCGR2A

MXD1

ALPL

LRRK2

BCL6

MMP25

LITAF

HERC5

APOBEC3A

MX2

FFAR2

FAM129A 
Interferon alpha/beta signaling Antiviral mechanism by IFN-stimulated genes

Interferon gamma signaling

Peptide chain elongation

Viral mRNA Translation nfluenza Infection

Eukaryotic Translation Elongation

Selenocysteine synthesis -

Eukaryotic Translation Termination

Nonsense Mediated Decay (NMD) independent of the Exon Junction Complex (EJC)

Formation of a pool of free $40 \mathrm{~S}$ subunits

L13a-mediated translational silencing of Ceruloplasmin expression SRP-dependent cotranslational protein targeting to membrane GTP hydrolysis and joining of the 605 ribosomal subunit Nonsense-Mediated Decay (NMD) Nonsense Mediated Decay (NMD) enhanced by the Exon Junction Complex (EJC)

Selenoamino acid metabolism

Eukaryotic Translation Initiation -

Cap-dependent Translation Initiation Influenza Viral RNA Transcription and Replication Influenza Life Cycle

Regulation of expression of SLITs and ROBOs Major pathway of rRNA processing in the nucleolus and cytosol

ISG15 antiviral mechanism

rRNA processing in the nucleus and cytosol rRNA processing

Infectious disease

Signaling by $\mathrm{ROBO}$ receptors

DDX58/IFIH1-mediated induction of interferon-alpha/beta Translation

Metabolism of amino acids and der ivatives

Negative regulators of DDX58/IFIH1 signaling -

TRAF3-dependent IRF activation pathway

Formation of the ternary complex, and subsequently, the 435 complex Translation initiation complex formation Ribosomal scanning and star $t$ codon recognition Activation of the mRNA upon binding of the cap-binding comple $x$ and elFs, and subsequent binding to $43 S$. TRAF6 mediated IRF7 activation ECM proteoglycans

$\mathrm{NF}-\mathrm{kB}$ activation through FADD/RIP-1 pathway mediated by caspase -8 and -10

GRB2:SOS provides linkage to MAPK signaling for Integrins p130Cas linkage to MAPK signaling for integrins Signal transduction by $L 1$

Platelet degranulation

Wieghted FastMix model with response and age

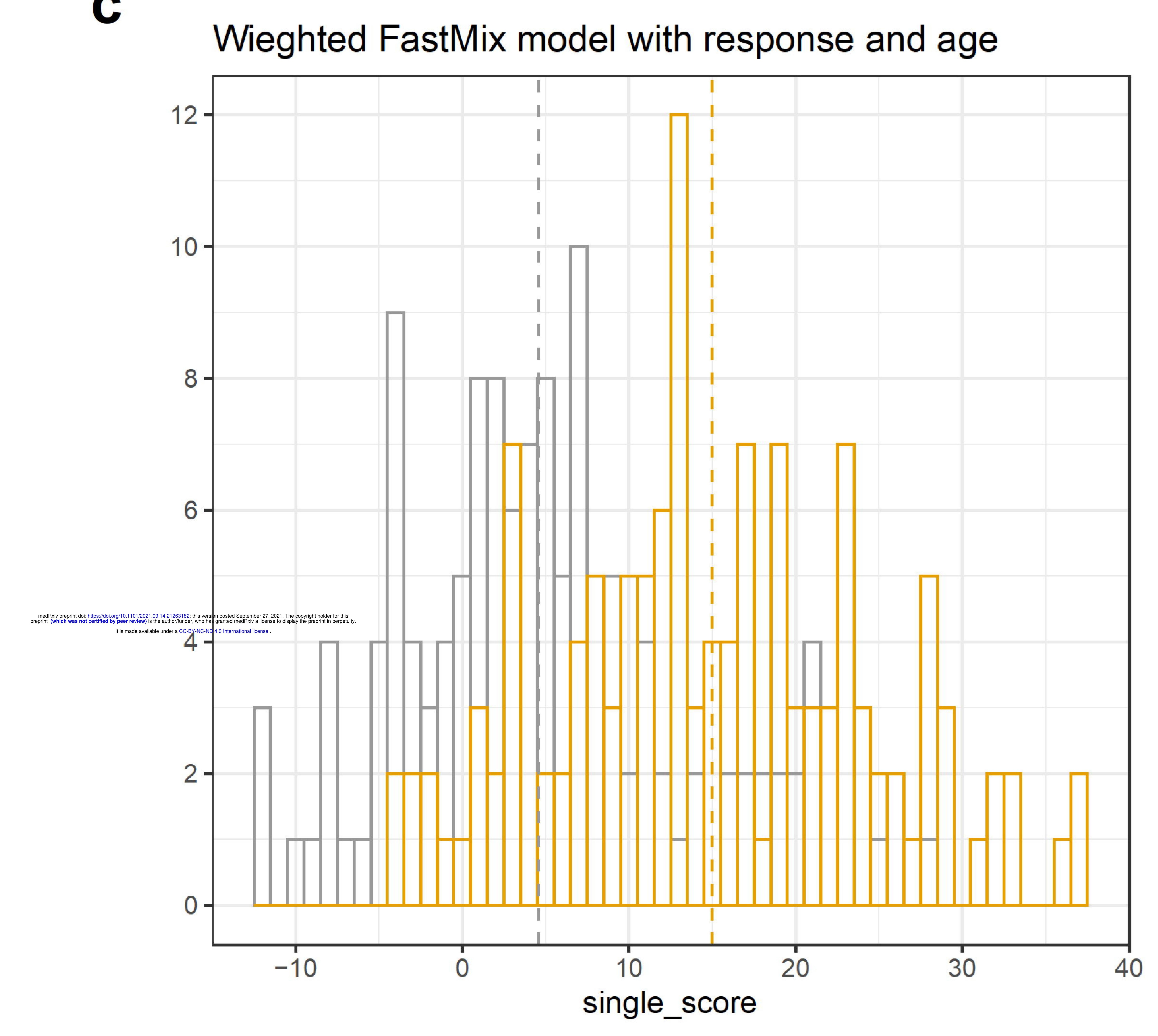

Wilcoxon $p$-value $=1.9 \mathrm{e}-16$ d

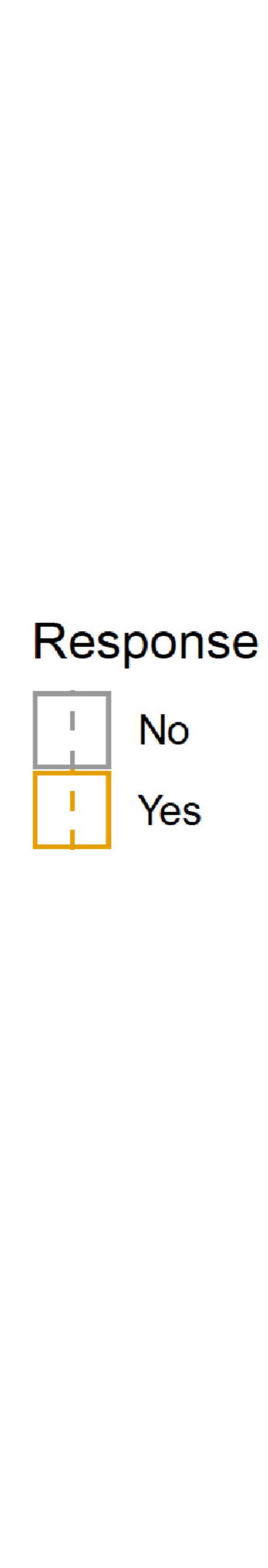

b CD45pCD66p.Response BST2

3 CHMP5

4 DDX58

5 DHX58

6 EIF2AK2

7 FCGR1B

8 GBP1

9 GBP3

10 GBP5

11 HERC5

12 IDO1

$13 \quad$ IFI35

$14 \quad$ IFI6

15 IFIH1

16 IFIT1

17 IFIT2

$\begin{array}{lll}\text { p.adjust } & 18 & \text { IFIT3 }\end{array}$

$\begin{array}{lll}19 & \text { IFITM3 }\end{array}$

20 IRF7

21 ISG15

22 ITGA2B

23 ITGB3

24 MT2A

25 MX1

26 OAS1

27 OAS2

28 OAS3

29 OASL

$30 \quad$ RPL23

$31 \quad$ RPL27

32 RPL31

33 RPL34

$34 \quad$ RPL39

35 RPL9

36 RPS15A

37 RPS3A

38 RPS4Y1

39 RPS7

40 RSAD2

41 SERPING1

42 SPARC

43 TRIM22

44 USP18

$45 \quad \mathrm{XAF} 1$
Wieghted FastMix model with response

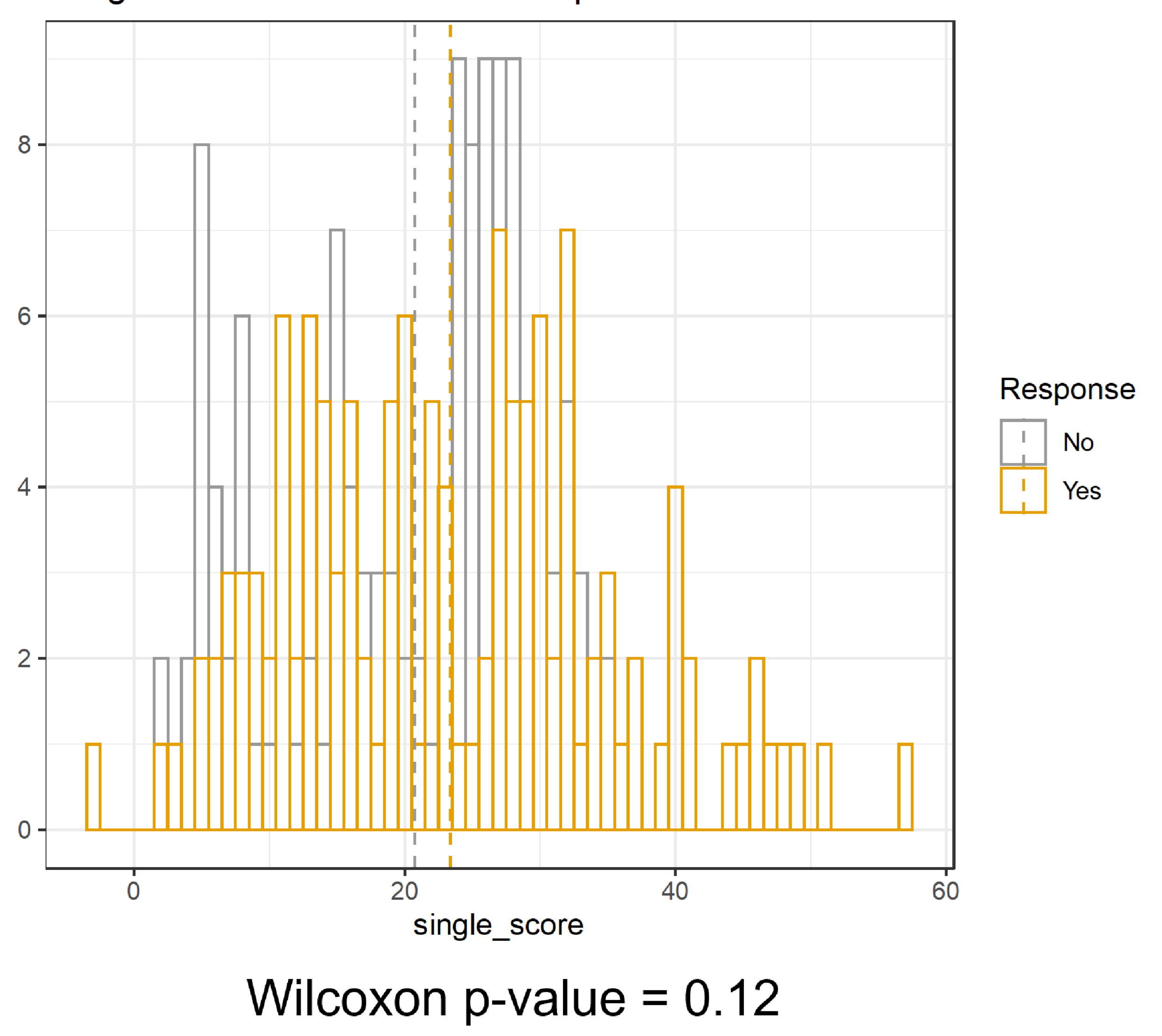


a
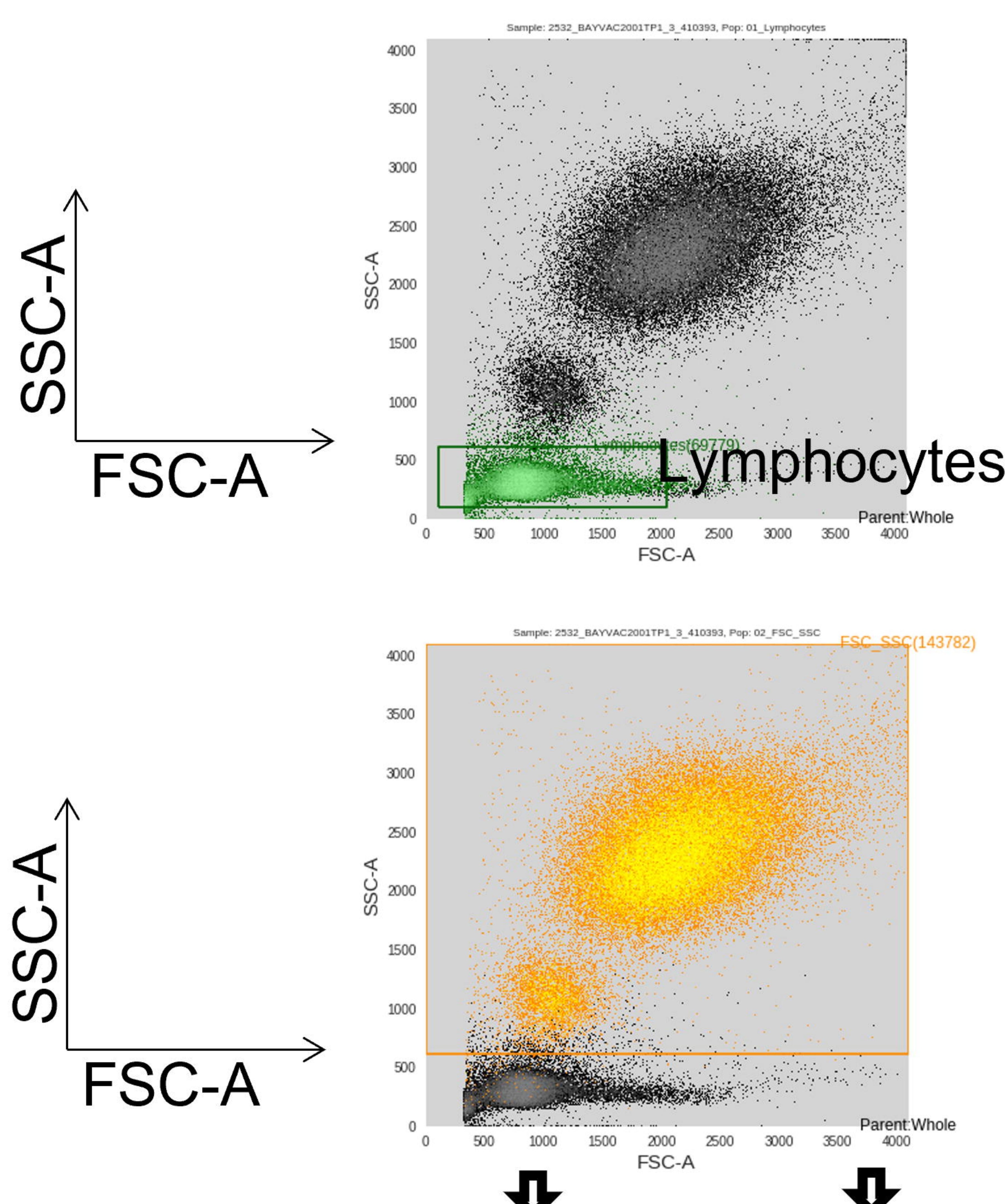

b Lymphocytes

Granulocytes

Monocytes

C
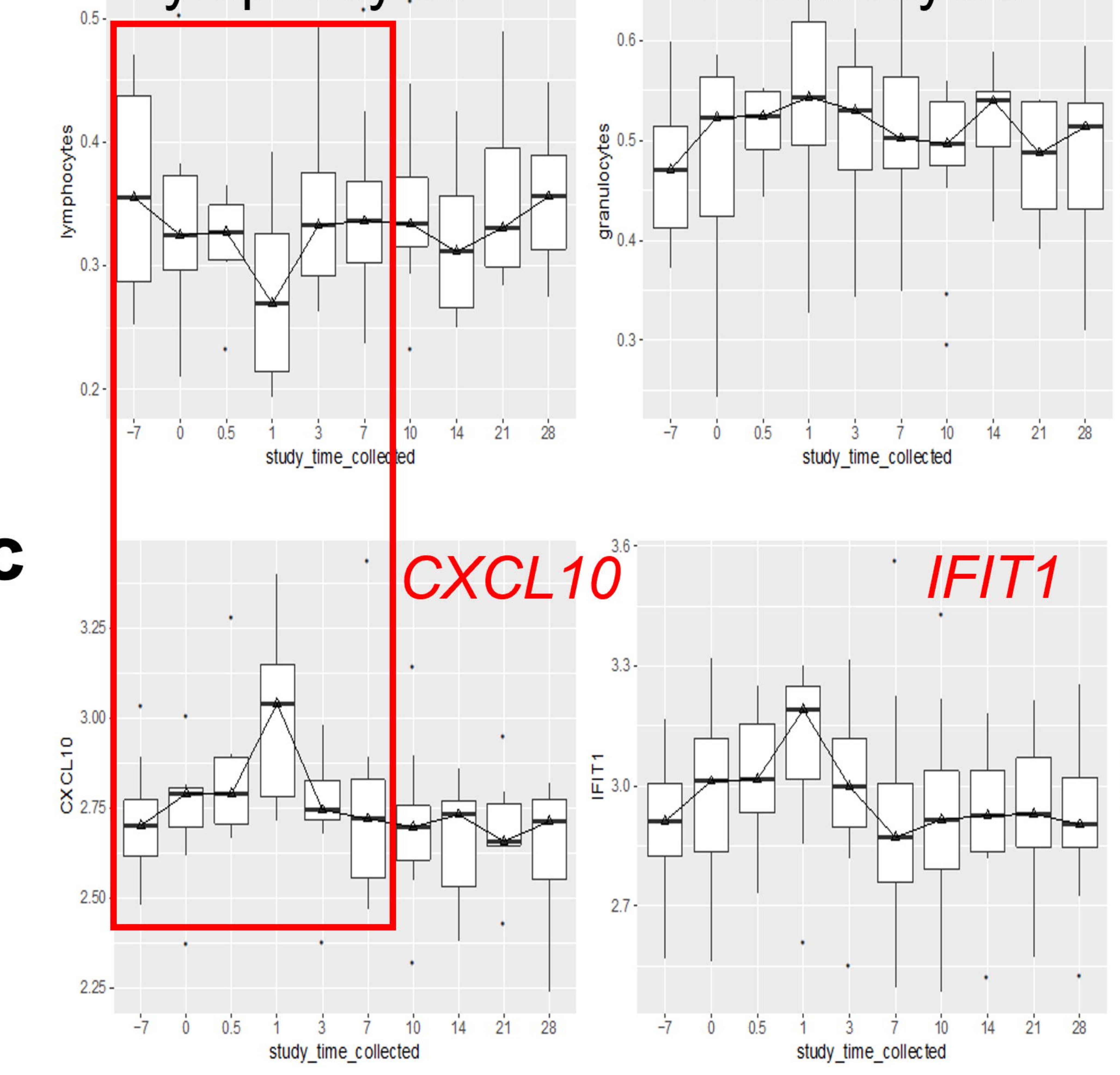

IFIT1

LAMP3

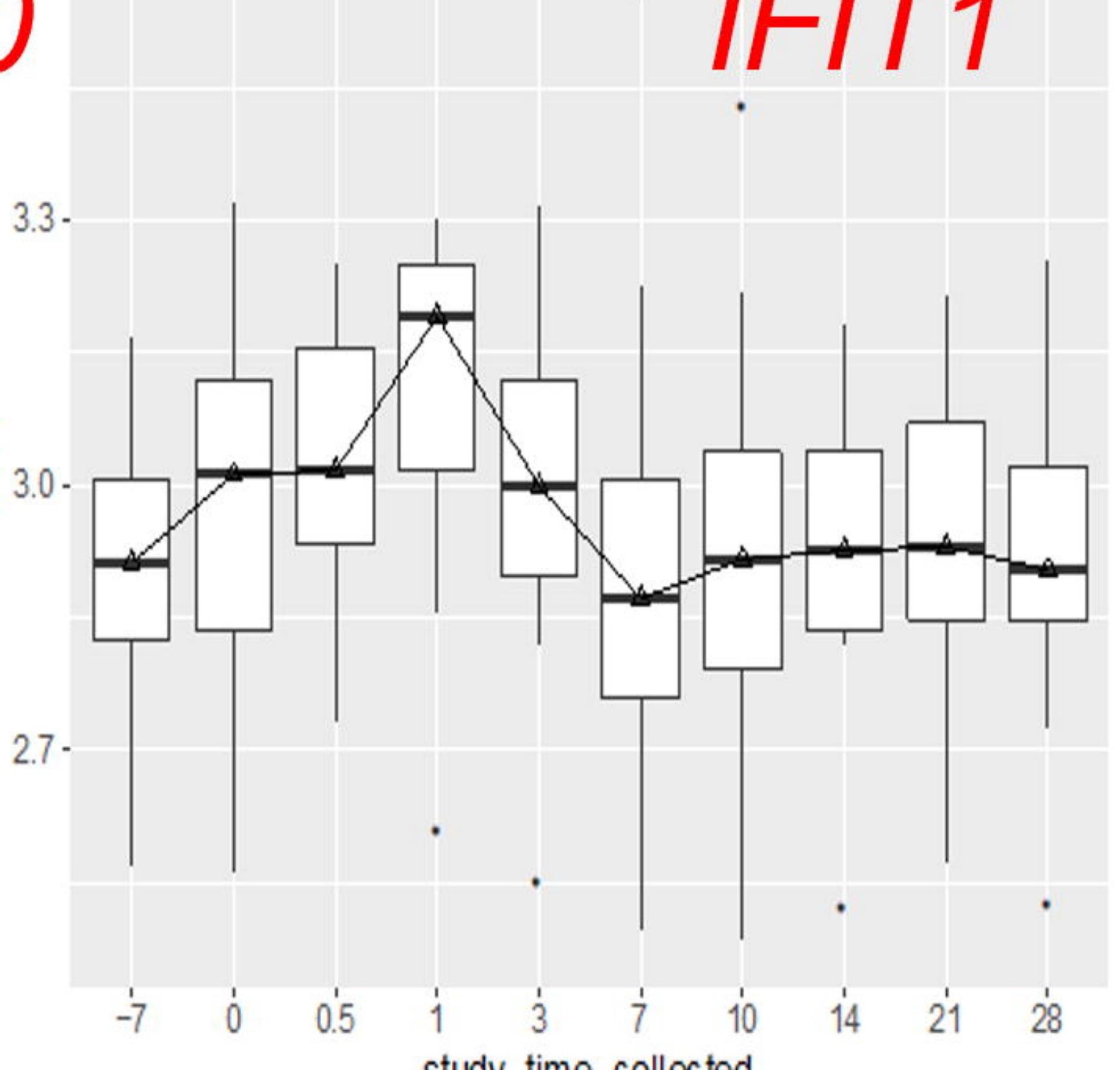

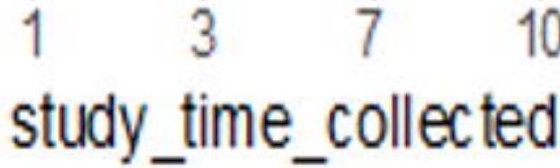

d

Lymphocyte

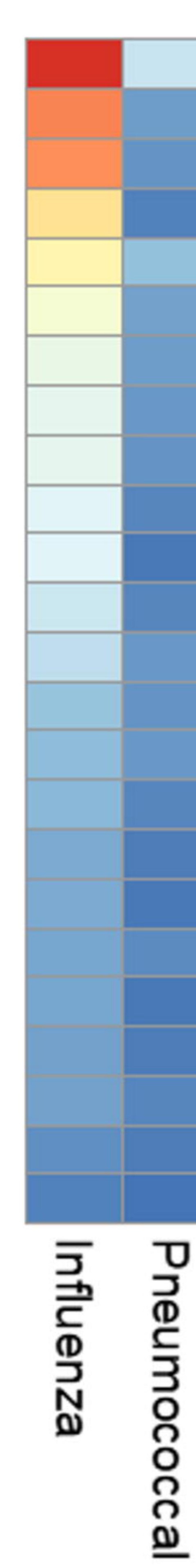

e Lymphocyte w.r.t. age

\begin{tabular}{|c|c|c|}
\hline 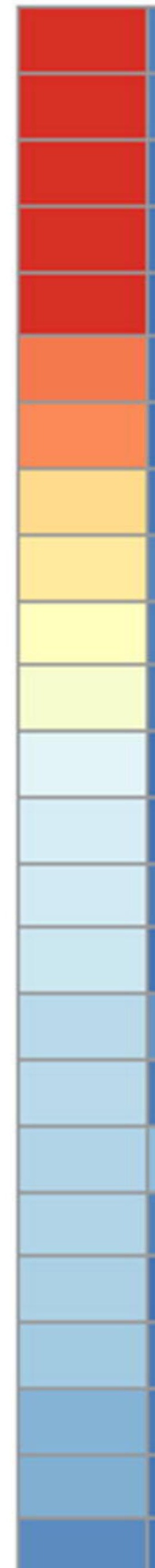 & & $\begin{array}{l}\text { BATF2 } \\
\text { LAMP3 } \\
\text { CXCL10 } \\
\text { SERPING1 } \\
\text { OASL } \\
\text { RSAD2 } \\
\text { IFIT3 } \\
\text { IFIT1 } \\
\text { TRIM6 } \\
\text { IFI44L } \\
\text { OAS3 } \\
\text { OAS1 } \\
\text { IFITM3 } \\
\text { EPSTI1 } \\
\text { ISG15 } \\
\text { LOC26010 } \\
\text { IFI44 } \\
\text { RTP4 } \\
\text { HERC5 } \\
\text { XAF1 } \\
\text { HES4 } \\
\text { MX1 } \\
\text { OAS2 } \\
\text { IY6F }\end{array}$ \\
\hline $\begin{array}{l}\bar{Z} \\
\stackrel{\vec{D}}{C} \\
\mathbb{D} \\
\stackrel{N}{N} \\
\mathbb{D}\end{array}$ & 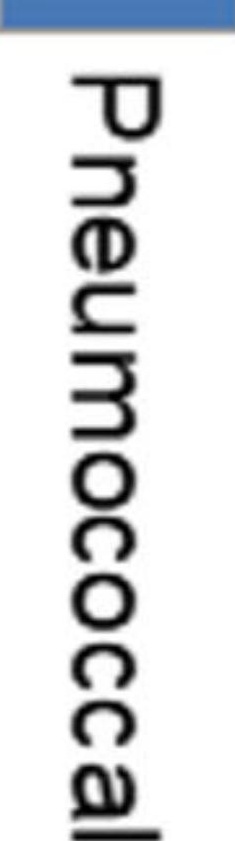 & \\
\hline
\end{tabular}




\begin{tabular}{|lll|ll|ll|ll|}
\hline \multicolumn{4}{|c|}{ No DEGs } & \multicolumn{3}{l|}{ With DEGs } \\
\hline & cor=0 & \multicolumn{2}{l|}{ cor=0.5 } & cor=0 & \multicolumn{2}{l|}{ cor=0.5 } \\
\hline Method & Time & MSE & Time & MSE & Time & MSE & Time & MSE \\
\hline lme4_ind & 1137.9 & 0.02 & 854.1 & 30.70 & 765.6 & 1.69 & 749.9 & 34.09 \\
lme4 & 8163.6 & 0.22 & 9798.9 & 0.32 & 8378.2 & 2.09 & 9525.6 & 1.96 \\
FastMix_ind & 27.9 & 0.04 & 27.6 & 34.3 & 29.4 & 0.49 & 28.7 & 34.57 \\
FastMix & 29.3 & 0.20 & 27.5 & 0.21 & 30.8 & 0.68 & 28.8 & 1.16 \\
\hline
\end{tabular}

\begin{tabular}{llll}
\hline cor $=0$ & Type-I Error & csSAM & FastMix \\
\hline & Cell1.Group & $28.99(11.19)$ & $6.36(1.11)$ \\
& Cell2.Group & $17.23(7.70)$ & $6.31(1.09)$ \\
& Cell3.Group & $13.05(7.61)$ & $5.04(0.21)$ \\
\hline cor $=0.5$ & Type-I Error & csSAM & FastMix \\
\hline & Cell1.Group & $17.34(9.11)$ & $6.85(0.42)$ \\
& Cell2.Group & $9.71(5.97)$ & $6.85(0.46)$ \\
& Cell3.Group & $6.86(5.54)$ & $5.00(0.23)$ \\
\hline
\end{tabular}

\begin{tabular}{lccc}
\hline MSE & $2.708(0.200)$ & $1.765(0.047)$ & $0.919(0.022)$ \\
\hline Cell1 & $-0.124(1.709)$ & $-2.127(1.821)$ & $-0.060(0.979)$ \\
Cell2 & $0.108(1.517)$ & $0.005(1.637)$ & $0.059(0.876)$ \\
Cell3 & $-0.102(1.520)$ & $-0.122(1.647)$ & $-0.124(0.876)$ \\
Severity & $-0.089(0.986)$ & $3.956(0.399)$ & $-0.081(0.618)$ \\
Sex & $-0.135(0.855)$ & $-0.087(0.360)$ & $-0.184(0.544)$ \\
Cell1.Severity & $0.015(1.912)$ & $-34.210(1.349)$ & $0.003(1.014)$ \\
Cell2.Severity & $0.252(2.107)$ & $9.125(1.438)$ & $0.212(1.238)$ \\
Cell3.Severity & $0.047(1.895)$ & $8.986(1.348)$ & $0.068(1.014)$ \\
Cell1.Sex & $-0.015(1.874)$ & $-0.308(1.291)$ & $0.183(0.994)$ \\
Cell2.Sex & $-0.015(2.078)$ & $0.063(1.390)$ & $-0.042(1.188)$ \\
Cell3.Sex & $0.287(1.866)$ & $0.298(1.293)$ & $0.218(0.996)$ \\
\hline
\end{tabular}
d $\begin{array}{llll}\text { cor }=0 & \text { Power } & \text { csSAM } & \text { FastMix }\end{array}$

\begin{tabular}{llll}
\hline & Cell1.Group & $56.48(17.75)$ & $61.86(4.11)$ \\
& Cell2.Group & $40.54(16.88)$ & $62.79(4.36)$ \\
\hline cor $=0.5$ & Power & csSAM & FastMix \\
\hline & Cell1.Group & $62.82(15.18)$ & $64.22(6.50)$ \\
& Cell2.Group & $46.72(14.88)$ & $64.68(6.31)$ \\
\hline
\end{tabular}

e Comp. Time csSAM FastMix

\begin{tabular}{lll}
\hline cor $=0$ & 209.05 & 20.82 \\
\hline cor $=0.5$ & 206.96 & 19.95 \\
\hline
\end{tabular}

